Algebraic 83 Geometric $\mathcal{T}_{\text {opology }}$

Volume 4 (2004) 543-569

Published: 15 July 2004

ATG

\title{
Automorphisms of free groups with boundaries
}

\author{
Craig A. Jensen \\ Nathalie Wahl
}

\begin{abstract}
The automorphisms of free groups with boundaries form a family of groups $\mathrm{A}_{n, k}$ closely related to mapping class groups, with the standard automorphisms of free groups as $\mathrm{A}_{n, 0}$ and (essentially) the symmetric automorphisms of free groups as $\mathrm{A}_{0, k}$. We construct a contractible space $L_{n, k}$ on which $\mathrm{A}_{n, k}$ acts with finite stabilizers and finite quotient space and deduce a range for the virtual cohomological dimension of $\mathrm{A}_{n, k}$. We also give a presentation of the groups and calculate their first homology group.
\end{abstract}

AMS Classification 20F65, 20F28; 20F05

Keywords Automorphism groups, classifying spaces.

\section{Introduction}

The group $A_{n, k}$ of automorphisms of free groups with $k$ boundaries was introduced in 29] to show that the natural map from the stable mapping class group of surfaces to the stable automorphism group of free groups gives an infinite loop map on the classifying spaces of the groups after plus construction. The "boundaries" make it possible to define appropriate gluing operations.

There are several ways to define these groups.

Geometrically, $\mathrm{A}_{n, k}$ is the group of components of the homotopy equivalences of a certain genus $n+k$ graph $G_{n, k}$ (see Figure 1) fixing $k$ circles in the graph. The mapping class group $\Gamma_{g, k+1}$ of a surface of genus $g$ with $k+1$ boundary components is in fact the subgroup of $\mathrm{A}_{2 g, k}$ fixing one more circle (see Section 21). An alternative geometric description is given in [1] in terms of the mapping class groups of a 3-dimensional manifold.

Algebraically, we first define a related group $\mathrm{A}_{n}^{k}$, which corresponds to mapping class groups with punctures rather than boundaries. Let $F_{n+k}$ denote the free group on the $n+k$ generators $x_{1}, \ldots, x_{n}, y_{1}, \ldots, y_{k}$, and let $\operatorname{Aut}\left(F_{n+k}\right)$ be its automorphism group. Then $\mathrm{A}_{n}^{k}:=\left\{\phi \in \operatorname{Aut}\left(F_{n+k}\right) \mid \phi\left(y_{i}\right) \sim y_{i}\right.$ for $\left.1 \leq i \leq k\right\}$, 
where $\sim$ means 'conjugate to'. The groups $\mathrm{A}_{n}^{k}$ are thus natural intermediates between $\mathrm{A}_{n}^{0}=\operatorname{Aut}\left(F_{n}\right)$ and $\mathrm{A}_{0}^{k}$ which is known as the pure symmetric automorphism group of $F_{k}$. The group $\mathrm{A}_{n, k}$ is a central extension

$$
1 \rightarrow \mathbb{Z}^{k} \rightarrow \mathrm{A}_{n, k} \rightarrow \mathrm{A}_{n}^{k} \rightarrow 1
$$

(see Section 2 for details).

The symmetric automorphisms of free groups have been studied extensively, in particular for their relation to automorphism groups of free products (see for example [1, 4, 14, 25, 26]) and for their relation to motion groups of strings [3, 6, 17, 26]; they give a generalization of the braid groups, which is the mapping class group of a genus 0 punctured surface. Our groups are the higher genus analogues, coming from surfaces with punctures for $\mathrm{A}_{n}^{k}$ or with boundaries for $\mathrm{A}_{n, k}$.

In [18, Section 4], Levitt defines a class of groups, which he calls relative automorphism groups, in order to study automorphism groups of hyperbolic groups and of graphs of groups. The groups $A_{n, k}$ are examples of relative automorphism groups.

In this paper, we construct a contractible space $L_{n, k}$ of dimension $2 n+3 k-2$ (provided $n \geq 1$; if $n=0$ it is of dimension $2 k-1$ ) on which the group $\mathrm{A}_{n, k}$ acts with finite stabilizers and such that the quotient space is a finite complex. The space $L_{n, k}$ is an analogue of the spine of Auter space $X_{n}$. Recall that $X_{n}$ is a space of graphs of genus $n$ marked by a rose $\vee_{n} S^{1}$. In $L_{n, k}$, we consider graphs of genus $n+k$ marked by the graph $G_{n, k}$ of Figure 1 We also construct an analogous space $L_{n}^{k}$ for the group $A_{n}^{k}$ so that there is a sequence of contractible spaces

$$
F_{n, k} \rightarrow L_{n, k} \rightarrow L_{n}^{k}
$$

corresponding to the group extension $\mathbb{Z}^{k} \rightarrow A_{n, k} \rightarrow A_{n}^{k}$. The fiber $F_{n, k}$ is homeomorphic to $\mathbb{R}^{k}$ with the standard $\mathbb{Z}^{k}$-action.

We use $L_{n, k}$ to give the following range for the virtual cohomological dimension of $\mathrm{A}_{n, k}: 2 n+2 k-1 \leq \operatorname{vcd}\left(\mathrm{A}_{n, k}\right) \leq 2 n+3 k-2$ when $k \geq 1$ (Theorem 3.5).

By the work of McCool 24, we know that $\mathrm{A}_{n}^{k}$ is finitely presented. We use his work to produce a presentation for $\mathrm{A}_{n}^{k}$ and $\mathrm{A}_{n, k}$ (Theorems 7.1 and [7.2). We recover the presentation he gave for $\mathrm{A}_{0}^{k}$ in [25]. Theorem [7.4] says that $H_{1}\left(A_{n, k}\right)=\mathbb{Z}_{2}$ when $n>2$. This agrees with the recently proved conjecture that the homology $H_{i}\left(\mathrm{~A}_{n, k}\right)$ is independent of $n$ and $k$ for $n>>i$ [1].

To show that $L_{n, k}$ is contractible, we show that it is isomorphic to a subcomplex of $X_{n+3 k}^{\Theta}$, the set of fixed points of $X_{n+3 k}$ under the action of a certain finite 
subgroup $\Theta$ of $\operatorname{Aut}\left(F_{n+3 k}\right)$. Although $\mathrm{A}_{n, k}$ is not a subgroup of $\operatorname{Aut}\left(F_{n+k}\right)$, it is a subgroup of the normalizer of $\Theta$ in $\operatorname{Aut}\left(F_{n+3 k}\right)$. Hence $\mathrm{A}_{n, k}$ acts on $X_{n+3 k}^{\Theta}$, which is known to be contractible [13. The space $L_{n+k}$ can be identified with

the essential realization of an initial segment of the essential realization of $X_{n+3 k}^{\Theta}$ under a certain norm, in the language of [17. Our Theorem 4.1] says that the initial segments of the essential realization of $X_{n}^{G}$ are contractible for any finite group $G \leqslant \operatorname{Aut}\left(F_{n}\right)$.

Our motivation is the study of the stable automorphism group Aut , $_{\infty}$, the colimit of the inclusions $\operatorname{Aut}\left(F_{n}\right) \hookrightarrow \operatorname{Aut}\left(F_{n+1}\right)$. The homology and homotopy type of the classifying space of the stable mapping class group $\Gamma_{\infty}$ has been determined recently [19, 20, 28, making use of the mapping class groups $\Gamma_{g, n}$ of surfaces with any number of boundary components, and the fact that the homology groups $H_{*}\left(\Gamma_{g, n}\right)$ are independent of $g$ and $n$ when $g>>* 8$. Little is known about $B$ Aut $_{\infty}$. It was shown recently that the homology of $\mathrm{A}_{n, k}$ is also independent of the number of boundaries in a stable range [11. This means in particular that we can work with $\mathrm{A}_{n, k}$ instead of $\operatorname{Aut}\left(F_{n}\right)$ if we are only interested in stable results. The boundaries of $\mathrm{A}_{n, k}$ allow us to define gluing operations analogous to the very useful gluing operations for the mapping class groups. These give a wealth of new operations on the automorphism of free groups and are used in 29] to show that the map on classifying spaces $B \Gamma_{\infty}^{+} \rightarrow B \mathrm{Aut}_{\infty}^{+}$is an infinite loop map after Quillen's plus-construction.

The paper is organized as follows. Section 2 recalls the definition of the groups $\mathrm{A}_{n, k}$ and describes their relationship to mapping class groups of surfaces. Section 3 defines the space $L_{n, k}$ and calculates a range for the virtual cohomological dimension of $\mathrm{A}_{n, k}$. In Section 4, we prove the contractibility of initial segments in a general setting. Section [5] shows that $L_{n, k}$ is an initial segment of $X_{n+3 k}^{\Theta}$. In Section [6] we exhibit the sequence $F_{n, k} \rightarrow L_{n, k} \rightarrow L_{n}^{k}$. Finally, Section 7 gives the presentations and calculates $H_{1}$.

\section{Acknowledgment}

The authors would like to thank Benson Farb for helpful conversations and the referee for interesting suggestions. The work was conducted while the first author was partially supported by Louisiana Board of Regents Research Competitiveness Subprogram Contract LEQSF-RD-A-39 and second was supported by a Marie Curie Fellowship of the European Community under contract number HPMF-CT-2002-01925. 


\section{The groups $\mathrm{A}_{n, k}$}

Let $G_{n, k}$ be the graph given in Figure 1 obtained from a wedge of $n$ circles by attaching $k$ circles $C_{1}, \ldots, C_{k}$ on stems. We think of the circles (together with the basepoint) as the boundary of the graph. The basepoint $*$ of $G_{n, k}$ is the central vertex. Define

$$
\mathrm{A}_{n, k}:=\pi_{0} \operatorname{Htpy}_{*}\left(G_{n, k} ; \partial\right),
$$

where $\operatorname{Htpy}_{*}\left(G_{n, k} ; \partial\right)$ is the space of (basepointed) homotopy equivalences of $G_{n, k}$ fixing the circles $C_{j}$ point-wise. By [9, Prop. 0.19], homotopy equivalences fixing the circles are actually homotopy equivalences relative to the circles. This shows in particular that $A_{n, k}$ is a group.

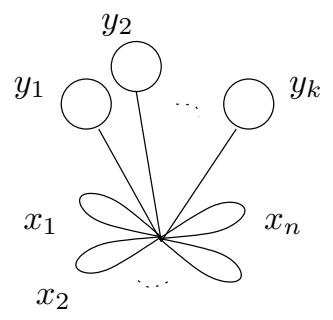

Figure 1: $G_{n, k}$

Labeling the first $n$ petals of $G_{n, k}$ with $x_{1}, \ldots, x_{n}$ and the $k$ circles with their stems $y_{1}, \ldots, y_{k}$ induces an identification of $\pi_{1}\left(G_{n+k}\right)$ with the free group $F_{n+k}:=\left\langle x_{1}, \ldots, x_{n}, y_{1}, \ldots, y_{k}\right\rangle$. This leads to the following algebraic description of $\mathrm{A}_{n, k}$ : Define a map $\alpha:\left(F_{n+k}\right)^{n+k} \rightarrow \operatorname{Hom}\left(F_{n+k}, F_{n+k}\right)$ by

$$
\begin{cases}\alpha\langle\nu, w\rangle\left[x_{i}\right]=\nu_{i} & 1 \leq i \leq n \\ \alpha\langle\nu, w\rangle\left[y_{j}\right]=w_{j}^{-1} y_{j} w_{j} & 1 \leq j \leq k\end{cases}
$$

where $\langle\nu, w\rangle:=\left(\nu_{1}, \ldots, \nu_{n}, w_{1}, \ldots, w_{k}\right) \in\left(F_{n+k}\right)^{n+k}$.

Proposition 2.1 There is a group isomorphism

$$
\mathrm{A}_{n, k} \cong\left\{\langle\nu, w\rangle \in\left(F_{n+k}\right)^{n+k} \mid \alpha\langle\nu, w\rangle \in \operatorname{Aut}\left(F_{n+k}\right)\right\}
$$

with the multiplication on the right hand side defined by

$$
\langle\nu, w\rangle \cdot\left\langle\nu^{\prime}, w^{\prime}\right\rangle=\left\langle\alpha\left\langle\nu^{\prime}, w^{\prime}\right\rangle[\nu], w^{\prime} . \alpha\left\langle\nu^{\prime}, w^{\prime}\right\rangle[w]\right\rangle,
$$

where we apply $\alpha\left\langle\nu^{\prime}, w^{\prime}\right\rangle$ component-wise, and the unit is $\left(x_{1}, \ldots, x_{n}, 1, \ldots, 1\right)$. 
Proof We use the labeling of $G_{n, k}$ by $x_{i}$ 's and $y_{j}$ 's as above (with a fixed orientation) to get an identification $\pi_{1}(G) \cong F_{n+k}$. The "lollies" $y_{j}$ are made out of a stem, denoted $l_{j}$, from the basepoint to the circle, denoted $C_{j}$.

For any $f \in \operatorname{Htpy}_{*}(G ; \partial)$, we want to define an element $\langle\nu, w\rangle$ as above which depends only on the homotopy class of $f$. Set $\nu_{i}:=\left[f\left(x_{i}\right)\right]$ and $w_{j}:=\left[l_{j} . f\left(\overline{l_{j}}\right)\right]$ in $\pi_{1}(G)$, where $\overline{l_{j}}$ means the path $l_{j}$ taken in the reverse direction. Now $\alpha\langle\nu, w\rangle$ is the automorphism induced by $f$ on $\pi_{1}(G)$. Indeed, $\left[f\left(x_{i}\right)\right]=\nu_{i}$ and $\left[f\left(y_{j}\right)\right]=\left[f\left(l_{j}\right) \cdot f\left(C_{j}\right) \cdot f\left(\overline{l_{j}}\right)\right]$ which is equal to $w_{j}^{-1} y_{j} w_{j}$ in $\pi_{1}(G)$. So $\langle\nu, w\rangle$ is an element of $\alpha^{-1}\left(\operatorname{Aut}\left(F_{n+k}\right)\right)$ as required.

Conversely, we want to show that any such $\langle\nu, w\rangle$ defines an element $[f] \in$ $\pi_{0} \operatorname{Htpy}_{*}(G ; \partial)$. Define $f$ piecewise by $f\left(x_{i}\right)=\nu_{i}, f\left(l_{j}\right)=w_{j}^{-1} . l_{j}$ and $f\left(C_{j}\right)=$ $C_{j}$. It is a continuous map, and a homotopy equivalence as it acts on $\pi_{1}(G)$ via $\alpha\langle\nu, w\rangle$.

The two maps are homomorphisms and inverse of one another. Hence the two groups are isomorphic.

The map $\alpha: \mathrm{A}_{n, k} \rightarrow \operatorname{Aut}\left(F_{n+k}\right)$ is a homomorphism (where the multiplication of automorphisms $\phi . \psi$ is defined by first applying $\phi$ and then $\psi$.) Geometrically, it is the map that forgets that the circles were fixed. It is not injective. There is in fact a short exact sequence

$$
1 \rightarrow \mathbb{Z}^{k} \stackrel{i}{\rightarrow} \mathrm{A}_{n, k} \stackrel{\alpha}{\rightarrow} \mathrm{A}_{n}^{k} \rightarrow 1
$$

where $\mathrm{A}_{n}^{k} \cong\left\{\phi \in \operatorname{Aut}\left(F_{n+k}\right) \mid \phi\left(y_{j}\right) \sim y_{j}\right.$ for $\left.1 \leq j \leq k\right\}$ and where $i\left(z_{1}, \ldots, z_{k}\right)=\left(x_{1}, \ldots, x_{n}, y_{1}^{z_{1}}, \ldots, y_{k}^{z_{k}}\right)$. Note that the group $\mathrm{A}_{n}^{k}$ can be described geometrically as $\pi_{0} \operatorname{Htpy}_{*}\left(G_{n, k} ;[\partial]\right)$, where $\operatorname{Htpy}_{*}\left(G_{n, k} ;[\partial]\right)$ is now the space of homotopy equivalences of $G_{n, k}$ fixing the circles up to a rotation.

The group $\mathbb{Z}^{k}$ maps to the center of $\mathrm{A}_{n, k}$. These $k$ additional generators in $\mathrm{A}_{n, k}$ correspond to "conjugating $y_{i}$ by itself". Geometrically, these are Dehn twists along the $i$ th boundary component in the corresponding mapping class group $\Gamma_{g, k+1}$.

We will also consider the semidirect product $A_{n, k}^{\Sigma}=\Sigma_{k} \ltimes \mathrm{A}_{n, k}$. Geometrically, $\mathrm{A}_{n, k}^{\Sigma}:=\pi_{0} \operatorname{Htpy}_{*}^{\Sigma}\left(G_{n, k} ; \partial\right)$ with $\operatorname{Htpy}_{*}^{\Sigma}\left(G_{n, k} ; \partial\right)$ the space of homotopy equivalences fixing the cycles $C_{j}$ up to a permutation. Algebraically, this means that the $y_{i}$ 's can be permuted.

The groups $\mathrm{A}_{n, k}$ and $\mathrm{A}_{n}^{k}$ are closely related to mapping class groups when $n$ is even. Indeed, note that the graph $G_{2 g, k}$ is homotopy equivalent to a surface $S_{g, k+1}$ of genus $g$ with $k+1$ boundary circles and $\mathrm{A}_{n, k}$ can also be defined as 
the group of components of the homotopy equivalences of $S_{g, k+1}$ fixing the first $k$ boundary components point-wise and a point on the last one.

Let $\Gamma_{g, k}:=\pi_{0} \operatorname{Diff}^{+}\left(S_{g, k+1} ; \partial\right)$ denote the mapping class group of $S_{g, k+1}$ with the boundaries point-wise fixed and let $\Gamma_{g}^{k+1}$ be the mapping class group of $S_{g, k+1}$ where the boundaries are fixed, but no longer point-wise. Fricke (1897) and Magnus (1934) for $g=0,1$ and Nielsen (1927) for $g \geq 2$ proved that $\Gamma_{g}^{k+1}$ is the subgroup of $\operatorname{Out}\left(F_{2 g+k}\right)$ fixing the cyclic words defined by the boundaries 21. p175].

Note that the inner automorphisms of $F_{n+k}$ form a normal subgroup of $\mathrm{A}_{n}^{k}$. Let $\mathrm{O}_{n}^{k}:=\mathrm{A}_{n}^{k} / \operatorname{Inn}\left(F_{n+k}\right)$. So $\mathrm{O}_{n}^{k}=\left\{\phi \in \operatorname{Out}\left(F_{n+k}\right) \mid \phi\left(y_{j}\right) \sim y_{j}\right.$ for $\left.1 \leq j \leq k\right\}$. It follows that $\Gamma_{g}^{k+1}$ is the subgroup of $\mathrm{O}_{n}^{k}$ fixing the conjugacy class of the last boundary component, that is the word $c:=\left[x_{1}, x_{2}\right] \ldots\left[x_{2 g-1}, x_{2 g}\right] y_{1} \ldots y_{k}$. One actually has the following isomorphism of short exact sequences:

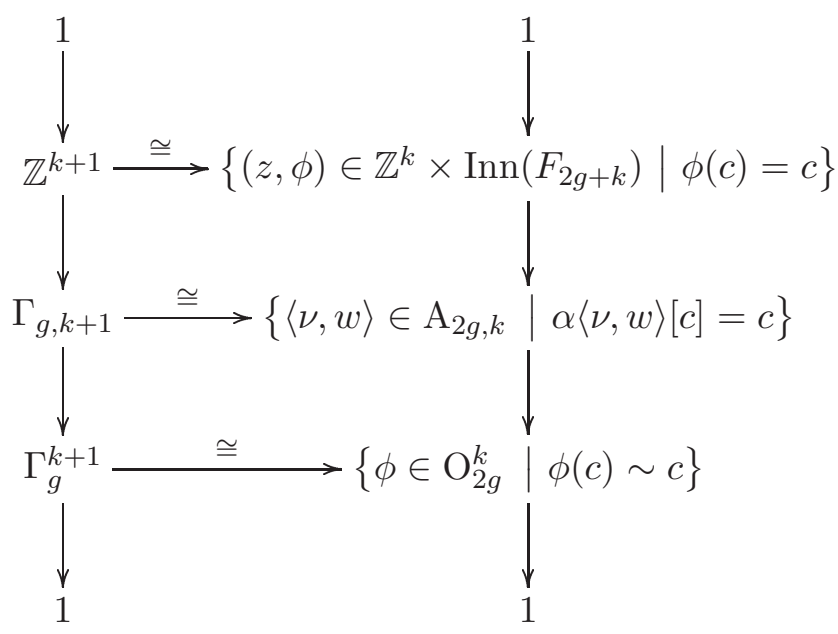

\section{$3(n, k)$-graphs}

By a graph, we mean a pointed, connected, one-dimensional CW-complex. Let $S^{1}$ be the circle with basepoint denoted by $\circ$.

Definition 3.1 An $(n, k)$-graph $(\Gamma, \underline{l})$ is a graph $\Gamma$ of genus $(n+k)$ with no separating edges, equipped with embeddings $l_{j}: S^{1} \rightarrow \Gamma$ for $j=1, \ldots, k$ such that

(1) For every $j=1, \ldots, k$, the point $\circ_{j}:=l_{j}(\circ)$ is a vertex of $\Gamma$. 
(2) The vertices of $\Gamma$ have valence at least 2 and if a vertex $v$ has valence 2 , then $v=\circ_{j}$ for some $j$ or $v$ is the basepoint $*$.

(3) Any two $\mathbb{S}_{j}:=l_{j}\left(S^{1}\right)$ meet in at most a point and the dual graph of the $\mathbb{S}_{j}$ 's is a forest.

We call the embedded circle $\left(\mathbb{S}_{j}, \circ_{j}\right)$ a cycle. In this way, an $(n, k)$-graph is a graph of genus $n+k$ with $k$ cycles. (See Figure 2 for an example.)

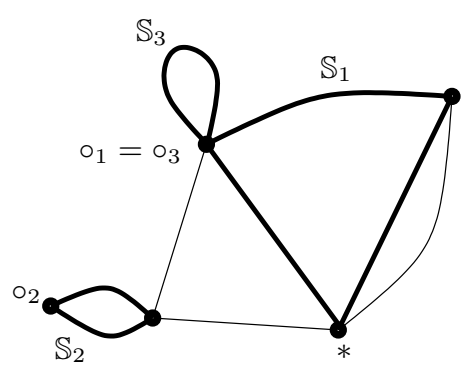

Figure 2: Example of a (2,3)-graph

Let $\left(G_{n, k}, \underline{\kappa}\right)$ be the generalized $(n, k)$-graph, where $G_{n, k}$ was defined in Section 2 (see Figure 1) and $\kappa_{j}: S^{1} \rightarrow G_{n, k}$ identifies $S^{1}$ with the $j$ th circle $C_{j}$. Note that $G_{n, k}$ has $k$ separating edges, and hence this is not an actual $(n, k)$-graph.

Definition 3.2 A marked $(n, k)$-graph $(\Gamma, \phi)$ is a graph $\Gamma$ equipped with a pointed homotopy equivalence $\phi: G_{n, k} \rightarrow \Gamma$ such that $(\Gamma, \phi \circ \underline{\kappa})$ is a $(n, k)$ graph.

Two marked $(n, k)$-graphs $\left(\Gamma^{1}, \phi^{1}\right)$ and $\left(\Gamma^{2}, \phi^{2}\right)$ are equivalent if there is a graph isomorphism $\psi: \Gamma^{1} \rightarrow \Gamma^{2}$ such that $\psi \circ \phi^{1}\left(C_{j}\right)=\phi^{2}\left(C_{j}\right)$ and $\psi \circ \phi^{1} \simeq \phi^{2}$ (rel. to $C_{j}$ for all $j$.)

The intuition (which is made precise in the proof of Theorem 5.3) is that condition (3) in Definition 3.1prevents the existence of Whitehead moves (blowing an edge and then collapsing it) which would take a 'good marking' to a 'bad marking'.

For an $(n, k)$-graph $(\Gamma, \underline{l})$, let $\hat{\Gamma}$ be the graph of genus $n$ obtained from $\Gamma$ by collapsing all the edges in the cycles $\mathbb{S}_{1}, \ldots, \mathbb{S}_{k}$. Let $e$ be an edge of $\Gamma$ which does not define a loop in $\Gamma$ or in $\hat{\Gamma}$. Then composition with the edge collapse $\Gamma \rightarrow \Gamma / e$ induces embeddings $l_{j} / e: S^{1} \rightarrow \Gamma / e$ such that $(\Gamma / e, \underline{l} / e)$ is again a $(n, k)$-graph. By an edge collapse in an $(n, k)$-graph, we will always mean the 
collapse of an edge satisfying the above hypothesis. For a marked $(n, k)$-graph, the marking of the collapsed graph is also obtained by composition with the collapse.

A forest collapse in a $(n, k)$-graph $(\Gamma, \underline{l})$ is a sequence of edge collapses. As the order of the collapses does not matter, it is defined by the union of the edges to be collapsed, which is a forest $F$ in $\Gamma$. We denote the collapsed graph by $(\Gamma / F, \underline{l} / F)$.

Let $L_{n, k}$ denote the set of equivalence classes of marked $(n, k)$-graphs. Define a poset structure on $L_{n, k}$ by saying that $\left(\Gamma^{1}, \phi^{1}\right) \leq\left(\Gamma^{2}, \phi^{2}\right)$ if there is a forest $F$ in $\Gamma^{2}$ such that $\left(\Gamma^{2} / F, \phi^{2} / F\right)$ is equivalent to $\left(\Gamma^{1}, \phi^{1}\right)$. By abuse of notation, we will also denote by $L_{n, k}$ the simplicial complex which is the geometric realization of this poset.

Using the geometric description of $A_{n, k}^{\Sigma}$, we obtain a natural action of $A_{n, k}^{\Sigma}$ (and thus of $\left.\mathrm{A}_{n, k}\right)$ on $L_{n, k}$ : For $\lambda \in A_{n, k}^{\Sigma}$ and $(\Gamma, \phi) \in L_{n, k}$, define

$$
\lambda \cdot(\Gamma, \phi):=\left(\Gamma, \phi \circ \lambda^{-1}\right) .
$$

We consider two equivalence relations on $(n, k)$-graphs: Two $(n, k)$-graphs $\left(\Gamma^{1}, \underline{l}^{1}\right)$ and $\left(\Gamma^{2}, \underline{l}^{2}\right)$ are $\Sigma$-equivalent if there is a graph isomorphism $\phi: \Gamma^{1} \rightarrow$ $\Gamma^{2}$ and a permutation $\sigma \in \Sigma_{k}$ such that $\phi \circ l_{j}^{1}(\circ)=l_{\sigma(j)}^{2}(\circ)$ and $\phi \circ l_{j}^{1} \simeq l_{\sigma(j)}^{2}$ (rel. to $\circ)$ for each $j=1, \ldots, k$. The $(n, k)$-graphs are equivalent if the permutation $\sigma$ is trivial.

Let $Q L_{n, k}^{\Sigma}$ and $Q L_{n, k}$ denote the set of all equivalence classes of $(n, k)$-graphs under each equivalence relation. Define spaces $Q L_{n, k}^{\Sigma}$ and $Q L_{n, k}$ by taking the elements of the sets as 0 -simplices and attaching a $p$-simplex for each equivalence class of chain of forests $\emptyset \neq F_{1} \subsetneq F_{2} \subsetneq \cdots \subsetneq F_{p}$, where two chains $F_{i}^{1}$ and $F_{i}^{2}$ are equivalent if there is a $(\Sigma$-)equivalence $\phi:(\Gamma, \underline{l}) \rightarrow(\Gamma, \underline{l})$ such that $\phi\left(F_{i}^{1}\right)=F_{i}^{2}$ for all $i=1, \ldots, p$. Note that these spaces are not simplicial complexes in general. The space $Q L_{n, k}$ is the moduli space of labeled $(n, k)$ graphs, that is $(n, k)$-graphs with cycles labeled $1, \ldots, k$, whereas in $Q L_{n, k}^{\Sigma}$ the cycles are unlabeled.

Proposition 3.3 The action of $A_{n, k}^{\Sigma}\left(\right.$ resp. $\left.\mathrm{A}_{n, k}\right)$ on $L_{n, k}$ is transitive on the sets of marked $(n, k)$-graphs with the same underlying unlabeled $(n, k)$-graph (resp. same underlying labeled $(n, k)$-graph), with finite simplex stabilizers, and the quotient of the action is $Q L_{n, k}^{\Sigma}$ (resp. $\left.Q L_{n, k}\right)$.

We will prove the following theorem in the next two sections.

Theorem 3.4 The space $L_{n, k}$ is contractible. 
We use this result to give a range for the virtual cohomological dimension of $\mathrm{A}_{n, k}$. We recall that it is already known that the ved of $\operatorname{Aut}\left(F_{n}\right)=\mathrm{A}_{n, 0}$ is

$2 n-2$ [5] and that the vcd of $\mathrm{A}_{0}^{k}$ is $k-1$ [4. Since $A_{n, k}$ is a subgroup of $\operatorname{Aut}\left(F_{n+3 k}\right)$ (see Proposition [5.1) and the latter group is virtually torsion free and of finite vcd, the former group is as well [2, Example 1 on page 229].

Theorem $3.5 \quad$ (1) $2 n+2 k-1 \leq \operatorname{vcd}\left(\mathrm{A}_{n, k}\right) \leq 2 n+3 k-2 \quad$ if $k \geq 1$.

(2) $\operatorname{vcd}\left(\mathrm{A}_{n, 1}\right)=2 n+1$.

(3) $\operatorname{vcd}\left(\mathrm{A}_{0, k}\right)=2 k-1$.

Proof of Theorem 3.5 For (1), we first note that there is a copy of $\mathbb{Z}^{2 n+2 k-1}$ in $\mathrm{A}_{n, k}$ : take $n$ generators sending $x_{i}$ to $x_{i} y_{k}$ and fixing all other generators, then $n$ generators sending $x_{i}$ to $y_{k} x_{i}$, then $k$ conjugating $y_{j}$ by $y_{k}$ for $1 \leq j \leq k$ and finally $k-1$ conjugating $y_{j}$ by $y_{j}$ for $j \neq k$.

For an upper bound, we calculate the dimension of a maximal simplex in $Q L_{n, k}$. Suppose the cycles lie in the maximally blown up graph in $c$ connected components and that the graph has $v$ vertices. The $k+1$ vertices $*, \circ_{1}, \ldots, \circ_{k}$ all have valence 2 . Since two cycles must meet in a valence 4 vertex and the dual graph of the cycles in the graph is a forest, there are $k-c$ vertices of valence 4 in the graph. The remaining vertices are valence 3 . Hence there are $e=3(v-2 k+c-1) / 2+(k+1)+2(k-c)=(3 v-c-1) / 2$ edges. Since $v-e=1-(n+k)$, this yields $v=2 n+2 k+c-1$ vertices. We can collapse these $v$ vertices down to 1 by first collapsing all except one edge in each cycle and then collapsing some remaining maximal tree. This yields a simplex of dimension $2 n+2 k+c-2$. Since $c \leq k$, the dimension of a maximal simplex is less than or equal to $2 n+3 k-2$. The result follows from Theorem 3.4.

For $(2)$, let $k=1$ in (1).

Finally for (3) if $n=0$ then $c=1$ and so $v=2 n+2 k+c-1=2 k$. Hence a maximal simplex has dimension $2 k-1$.

In Figure 3] we show an example of a maximal simplex of $Q L_{1,2}$ given by a graph with 2 connected cycle components and a forest consisting of $6>5$ edges.

\section{Initial segments of fixed point subcomplexes}

Recall from [10] that the spine of Auter space $X_{n}$ is a poset of pointed marked graphs of genus $n$, where the marking is given by a map from the rose $R_{n}:=$ 


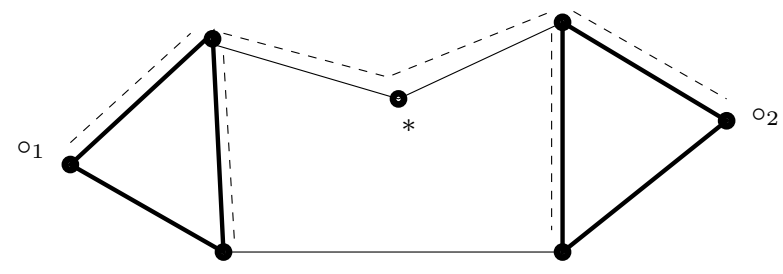

Figure 3: A maximal simplex in $Q L_{1,2}$ with a 6 edge forest

$\vee_{n} S^{1} . X_{n}$ is contractible and admits an action of $\operatorname{Aut}\left(F_{n}\right)$. Let $G$ be a finite subgroup of $\operatorname{Aut}\left(F_{n}\right)$ and consider the fixed point subcomplex $X_{n}^{G}$. A point in $X_{n}^{G}$ is a marked graph with a $G$-action. $X_{n}^{G}$ is contractible [13, Thm. 1.1].

Edge collapses induce a poset structure on $X_{n}^{G}$ with minimal elements the reduced marked graphs, i.e. those with no $G$-invariant subforest. An edge $e$ of a marked $G$-graph $\Gamma$ is essential if there exists a maximal $G$-invariant forest which does not contain $e$. The graph $\Gamma$ is essential if all of its edges are essential. Let $E_{n}^{G}$ be the full subcomplex of $X_{n}^{G}$ spanned by essential marked graphs. Collapsing inessential edges induces a poset map $f: X_{n}^{G} \rightarrow E_{n}^{G}$ such that $f(x) \leq x$ for all $x$ in $X_{n}^{G}$, and hence a deformation retraction on the corresponding spaces by the Poset Lemma (see [27] or [4, Lem. 4.1] for a direct proof of this special case).

Let $\mathcal{W}$ be the set of conjugacy classes of elements of $F_{n}$. Recall from 13, Section 2] that, given any well ordering of $\mathcal{W}$ and of $F_{n}$, we can define a norm

$$
\|\cdot\|_{\text {tot }}=\|\cdot\|_{\text {out }} \times\|\cdot\|_{\text {aut }} \in \mathbb{Z}^{\mathcal{W}} \times \mathbb{Z}^{F_{n}}
$$

on $E_{n}^{G}$ which well orders the reduced marked graphs of $E_{n}^{G}$ (using the corresponding lexicographic ordering). For $w \in \mathcal{W}$, the $w$ th component of the norm is the sum of the cyclic lengths of the paths $\phi(x w)$ for all $x \in G$, where the cyclic length is the number of edges in the path reduced cyclically, i.e. the length of the shortest path in its unpointed homotopy class. And for $z \in F_{n}$, the $z$ th component of the norm is the sum of the lengths of the paths $\phi(x z)$ for all $x \in G$, where we now consider the shortest paths in the pointed homotopy classes.

A nonempty set $\Lambda$ of reduced marked graphs of $E_{n}^{G}$ is called an initial segment if whenever two reduced marked graphs $r_{1}, r_{2}$ satisfy $\left\|r_{1}\right\|_{t o t} \leq\left\|r_{2}\right\|_{t o t}$ and $r_{2} \in \Lambda$, then $r_{1} \in \Lambda$. The realization of an initial segment $\Lambda$ is the space

$$
\|\Lambda\|=\bigcup_{r \in \Lambda} s t(r)
$$


the union of the ascending stars (in $E_{n}^{G}$ ) of the reduced marked graphs in $\Lambda$.

Let $\Lambda$ be an initial segment and let $\Gamma$ be a marked graph in $\|\Lambda\|$. An edge $e$ of $\Gamma$ is essential in $\Lambda$ if there is a maximal $G$-invariant subforest $\mathcal{F}$ of $\Gamma$ such that $e \notin \mathcal{F}$ and $\Gamma / \mathcal{F} \in \Lambda$. The graph $\Gamma$ is essential in $\Lambda$ if all of its edges are essential in $\Lambda$. The essential realization of an initial segment $\Lambda$ is the full subcomplex $|\Lambda|$ of $\|\Lambda\|$ spanned by the essential marked graphs of $\Lambda$.

Let $\Gamma$ be a marked graph in $E_{n}^{G}$ and let $v$ be a vertex of $\Gamma$. An ideal edge $\gamma$ at the vertex $v$ is a set of half edges of $\Gamma$ terminating at $v$ such that the blow-up $\Gamma^{\gamma}$ is again in $E_{n}^{G}$, where $\Gamma^{\gamma}$ is the graph obtained by $G$-equivariantly pulling the half edges in $\gamma$ away from $v$ (see [17, Section 5.A.] and [13, Section 2] for a characterization of ideal edges when $\Gamma$ is reduced.) More precisely, one creates a new edge orbit $G \gamma$ and a new vertex orbit $G v(\gamma)$. In $\Gamma^{\gamma}, \gamma$ is an actual edge that goes from $v(\gamma)$ to $v$. In addition, each half edge $e \in \gamma$ is now attached to $v(\gamma)$ instead of $v$, and this process is done equivariantly on $G \gamma$. Note that the original graph $\Gamma$ can be recovered from its blow up $\Gamma^{\gamma}$ by collapsing $G \gamma$.

An ideal forest in a reduced marked graph is a sequence of ideal edges satisfying certain technical conditions, which make sure that by blowing up these ideal edges, one can construct a blow-up $\Gamma^{\mathcal{F}}$ in the star of $\Gamma$ with an actual $G$ equivariant forest, whose partial collapses contain all the blow-ups of the ideal edges in the ideal forest. One can define a poset structure on the set of ideal forests of a reduced marked graph such that blowing up induces an isomorphism between this poset and the star of $\Gamma$ in $E_{n}^{G}$ [17, Prop. 5.9].

Let $\gamma$ be an ideal edge of a reduced $G$-graph $\Gamma$. Define $D(\gamma)$ to be the set of edges $c$ of $\gamma$ such that $G c$ is a forest in $\Gamma^{\gamma}$. To a pair $(\gamma, c)$ with $c \in D(\gamma)$ corresponds a Whitehead move that consists of first blowing up $\gamma$ and then collapsing Gc. An ideal edge $\gamma$ is reductive if there is a $c \in D(\gamma)$ such that performing the Whitehead move $(\gamma, c)$ yields a graph of smaller norm.

Let $Y$ be any of $E_{n}^{G},\|\Lambda\|$ or $|\Lambda|$. The reductive link of a reduced marked graph $\Gamma$ in $Y$ is the part of the star of $\Gamma$ in $Y$ spanned by ideal forests with at least one reductive ideal edge. The purely reductive link of $\Gamma$ in $Y$ is the part of the star of $\Gamma$ in $Y$ spanned by nontrivial ideal forests with all edges reductive. By the Poset Lemma, there is a deformation retraction from the reductive link of $\Gamma$ to the purely reductive link of $\Gamma$ given by collapsing non-reductive ideal edges.

Theorem 4.1 If $\Lambda$ is an initial segment of $E_{n}^{G}$, then both its realization $\|\Lambda\|$ and its essential realization $|\Lambda|$ are contractible. 
Proof By the Poset Lemma, collapsing inessential edges induces a deformation retraction from $\|\Lambda\|$ to $|\Lambda|$. Thus it is enough to show that $\|\Lambda\|$ is contractible.

If $r$ is a reduced marked graph in $\|\Lambda\|$, we have already observed that the reductive link of $r$ in $\|\Lambda\|$ deformation retracts onto the purely reductive link of $r$ in $\|\Lambda\|$. Since $\Lambda$ is an initial segment, the purely reductive link of $r$ in $\|\Lambda\|$ is the purely reductive link of $r$ in $E_{n}^{G}$. From [13, Lem. $3.1-3.7$ ], this reductive link (referred to as $S(\mathcal{R})$ in [13]) is contractible. As $\Lambda$ is an initial segment, all $\Gamma^{\prime}$ such that $\left\|r^{\prime}\right\|<\|r\|$ are also in $\Lambda$. The reductive link of a reduced marked graph $r$ is the intersection

$$
\operatorname{red}(r)=s t_{\|\Lambda\|}(r) \cap \bigcap_{\left\|r^{\prime}\right\|<\|r\|} s t_{\|\Lambda\|}\left(r^{\prime}\right) .
$$

Now the norm well orders the reduced marked graphs in $\|\Lambda\|$. We can build $\|\Lambda\|$ as the union of the stars of the elements of $\Lambda$, starting with the star of the smallest element and adding the stars successively. As the star of a graph is contractible and all the successive intersections are contractible, transfinite induction implies that every connected component of $\|\Lambda\|$ is contractible.

It remains to show that $\|\Lambda\|$ is connected. From [16. Thm. 2], we know that any two reduced marked graphs in $E_{n}^{G}$ are connected by a sequence of Whitehead moves. The reduced marked graphs of $E_{n}^{G}$ are ordered by the norm. Choose $I \subseteq \mathbb{Z}^{\mathcal{W}} \times \mathbb{Z}^{F_{n}}$ such that the ordered set of reduced marked graphs of $E_{n}^{G}$ is $\left\{r_{i}: i \in I\right\}$ and denote by $r_{0}$ the least element. Note that $r_{0} \in \Lambda$ because $\Lambda$ is nonempty. Note also that the stars $s t_{\|\Lambda\|}\left(r_{i}\right)$ and $s t_{E_{n}^{G}}\left(r_{i}\right)$ of $r_{i}$ in $\|\Lambda\|$ and $E_{n}^{G}$ are equal. We will denote these stars by $s t\left(r_{i}\right)$.

Suppose that $\|\Lambda\|$ is not connected. The star $s t\left(r_{0}\right)$ is contractible and thus connected. Take the least index $j$ such that $\cup_{i \leq j} s t\left(r_{i}\right)$ is not connected. Then we can get from $r_{0}$ to any $r_{i}$ with $i<j$ in $\|\Lambda\|$, but not from $r_{0}$ to $r_{j}$. Since $E_{n}^{G}$ is connected and $I$ is a well ordering, we can choose a least $k$ such that there is a path from $r_{0}$ to $r_{j}$ in $\cup_{i \leq k} s t\left(r_{i}\right)$. This implies that the reductive link of $r_{k}$ is not connected, however, since it has at least two components: one coming from $r_{0}$ and the other coming from $r_{j}$. This contradicts the fact that the reductive link is contractible. So $\|\Lambda\|$ is connected.

\section{Contractibility of $L_{n, k}$}

In this section, we prove Theorem 3.4 by identifying $L_{n, k}$ with the essential realization of an initial segment and applying Theorem 4.1. 
Let $m=n+3 k$. Let $\left\{x_{1}, \ldots, x_{n}, y_{1}, \ldots, y_{k}, u_{1}, \ldots, u_{k}, v_{1}, \ldots, v_{k}\right\}$ denote the generators of $F_{m}$. For each $j=1, \ldots, k$, let $\Theta_{j} \cong \mathbb{Z} / 3$ be the subgroup of $\operatorname{Aut}\left(F_{m}\right)$ generated by $\theta_{j}$, where $\theta_{j}\left(u_{j}\right)=v_{j}, \theta_{j}\left(v_{j}\right)=v_{j}^{-1} u_{j}^{-1}$, and all other generators are fixed. This group can be realized as the group of rotations of the three edges of a theta-graph (see Figure 5). Let

$$
\Theta=\Theta_{1} \times \Theta_{2} \times \cdots \times \Theta_{k} .
$$

be the direct product of these $k$ groups. The normalizer $N_{\text {Aut }\left(F_{m}\right)}(\Theta)$ acts on the fixed point subcomplex $X_{m}^{\Theta}$ and thus on $E_{m}^{\Theta}$. Both spaces are contractible [13] (see also Section 4).

Note that a similar fixed point subcomplex was used in [15] to study the holomorph of free groups $F_{n} \rtimes \operatorname{Aut}\left(F_{n}\right)$. This last group is the subgroup of $\mathrm{A}_{n}^{1}$ of automorphisms where $y_{1}$ does not occur in the images of the $x_{i}$ 's. One could work with circles and a $\mathbb{Z} / 2$-action, instead of $\Theta$-graphs and a $\mathbb{Z} / 3$-action. This would however bring a few extra technical problems (having to introduce extra vertices to avoid acting on edges by inversions) while producing the same complex. (See also Section [6])

Although $\mathrm{A}_{n, k}$ is not a subgroup of $\operatorname{Aut}\left(F_{n+k}\right)$, it is a subgroup of $\operatorname{Aut}\left(F_{n+3 k}\right)$ :

Proposition 5.1 There is an inclusion of groups $\beta: A_{n, k}^{\Sigma} \hookrightarrow N_{\operatorname{Aut}\left(F_{n+3 k}\right)}(\Theta) \leqslant$ $\operatorname{Aut}\left(F_{n+3 k}\right)$.

Proof The map $\beta$ is induced geometrically by attaching two additional circles at the end of each of the $k$ stems (see Figure 4). Algebraically, this map can be described as follows: for $\left\langle\sigma, \nu_{i}, w_{j}\right\rangle \in A_{n, k}^{\Sigma}=\Sigma_{k} \rtimes A_{n, k}$,

$$
\phi=i\left\langle\sigma, \nu_{i}, w_{j}\right\rangle \operatorname{maps}\left\{\begin{aligned}
x_{i} & \rightarrow \nu_{i} \\
y_{j} & \rightarrow w_{j}^{-1} y_{\sigma(j)} w_{j} \\
u_{j} & \rightarrow w_{j}^{-1} u_{\sigma(j)} w_{j} \\
v_{j} & \rightarrow w_{j}^{-1} v_{\sigma(j)} w_{j}
\end{aligned}\right.
$$

One then checks easily that $\phi=\beta\left\langle\sigma, \nu_{i}, w_{j}\right\rangle$ satisfies $\phi \theta_{l} \phi^{-1}=\theta_{\sigma(l)}$ for each $l=1, \ldots, k$.

For each $j=1, \ldots, k$, let $\theta^{j}$ denote a graph with two vertices $\circ_{j}$ and $v_{j}$, and three edges $e_{1}^{j}, e_{2}^{j}, e_{3}^{j}$ where each $e_{i}^{j}$ goes from $\circ_{j}$ to $v_{j}$ (See Figure [5). Consider the subcomplex Fix of $X_{m}$ spanned by marked graphs $\phi: R_{n} \rightarrow \tilde{\Gamma}$ which satisfy the following conditions: 


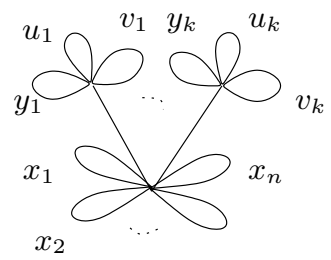

Figure 4: Graph giving the inclusion $\mathrm{A}_{n, k} \hookrightarrow \operatorname{Aut}\left(F_{n+3 k}\right)$

- The graph $\tilde{\Gamma}$ is obtained from a graph $\Gamma$ of genus $n+k$ by attaching the graphs $\theta^{1}, \ldots, \theta^{k}$ to $\Gamma$, identifying the vertex $\circ_{j}$ of $\theta^{j}$ with a vertex of $\Gamma$ and remembering these - possibly multiple - labels.

- For each $j=1, \ldots, k$, there exists a path $w_{j}$ in $\tilde{\Gamma}$ from $o_{j}$ to $*$ such that

$$
\phi\left(u_{j}\right)=\bar{w}_{j} \star e_{1}^{j} \star \bar{e}_{2}^{j} \star w_{j}
$$

and

$$
\phi\left(v_{j}\right)=\bar{w}_{j} \star e_{2}^{j} \star \bar{e}_{3}^{j} \star w_{j}
$$

where $\bar{p}$ denote the path $p$ with the reverse orientation and $\star$ denotes the concatenation of paths.
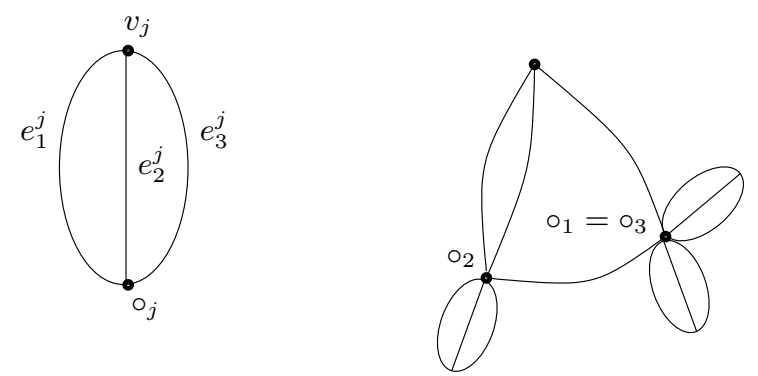

Figure 5: The graph $\theta^{j}$ and a graph of Fix

Proposition $5.2 E_{m}^{\Theta} \cong F i x \cong X_{m}^{\Theta}$.

Proof Each marked graph in Fix is fixed by the action of $\Theta$ as $\theta_{j}$ cyclically rotates the edges of the attached $\theta^{j}$. So Fix $\subset X_{m}^{\Theta}$.

Let $R_{n, k}^{\Theta}$ be the graph obtained by wedging the $k$ graphs $\theta^{j}$ to the rose $R_{n+k}$. Recall that a graph is reduced if it has no invariant subforest. Note that all the reduced graphs in Fix have the same underlying graph $R_{n, k}^{\Theta}$. 
Krstić showed that any two reduced $\Theta$-graphs in $X_{m}^{\Theta}$ are connected by a sequence of Whitehead moves $(\{e, f\}, f)$ where $e, f$ have the same terminal vertex and $\operatorname{Stab}_{\Theta}(e) \subseteq \operatorname{Stab}_{\Theta}(f)$ (and $e \notin \Theta f$ ) [16. Prop. 4']. Pictorially the move $(\{e, f\}, f)$ corresponds to pulling each edge in $\Theta e$ along the corresponding edge in $\Theta f$. Because of the condition on stabilizers, we can only perform Whitehead moves on $R_{n, k}^{\Theta}$ of the form $(\{e, f\}, f)$ or $\left(\left\{\bar{e}_{i}^{j}, f\right\}, f\right)$ where $e, f$ are edges of $R_{n+k}$ and $\bar{e}_{i}^{j}$ is in $\theta^{j}$. The first type of moves affect only the first $n+k$ petals, whereas the second type takes the subgraph $\theta^{j}$ around the edge $f$. The reduced graphs in Fix are closed under these moves.

Hence the reduced graphs in $X_{m}^{\Theta}$ are exactly the reduced graphs in Fix. Now the ascending star of a reduced graph in Fix is the same as the ascending star of the graph in $X_{m}^{\Theta}$ as equivariant blow-ups preserve the $\theta$-graphs (see [12, Claim 6.6] for a similar computation). So Fix $=X_{m}^{\Theta}$. Note finally that all graphs in Fix are essential as no edge in a $\theta^{j}$ can be collapsed $\Theta$-equivariantly and the other edges, being non-separating by hypothesis, are thus also essential. So $F i x=X_{m}^{\Theta}=E_{m}^{\Theta}$.

We now choose a well ordering of $\mathbb{Z}^{\mathcal{W}} \times \mathbb{Z}^{F_{n}}$ such that the the space $L_{n, k}$ of marked $(n, k)$-graphs is the realization of an initial segment of the induced norm $\|\cdot\|_{t o t}$. Well order the set of conjugacy classes $\mathcal{W}$ so that its first $k$ elements are

$$
u_{1} y_{1} u_{1}, u_{2} y_{2} u_{2}, \ldots, u_{k} y_{k} u_{k}
$$

and well order $F_{n}$ in any manner. Now define $\Lambda$ to be the set of reduced $\Theta$ graphs in $E_{m}^{\Theta}$ whose norm is minimal in the first $k$ coordinates. This is an initial segment in the lexicographic ordering induced by the norm. Recall that all reduced marked graphs in $E_{m}^{\Theta}$ have underlying graph $R_{n, k}^{\Theta}$. The minimal norm on the component $u_{j} y_{j} u_{j}$ is thus $(2+1+2) \cdot|\Theta|=5 \cdot 3^{k}$. The marking $\phi$ of such a minimal reduced marked graph is determined on $y_{j}, u_{j}$ and $v_{j}$ by a loop $w_{j}$ at $*$ and a single edge loop $\mathbb{S}_{j}$ at $*$ in the graph as follows:

$$
\begin{aligned}
& \phi\left(y_{j}\right)=\bar{w}_{j} \star \mathbb{S}_{j} \star w_{j} \\
& \phi\left(u_{j}\right)=\bar{w}_{j} \star e_{1}^{j} \star \bar{e}_{2}^{j} \star w_{j} \\
& \phi\left(v_{j}\right)=\bar{w}_{j} \star e_{2}^{j} \star \bar{e}_{3}^{j} \star w_{j},
\end{aligned}
$$

Note that the edge loops $\mathbb{S}_{j}$ have to be different for $\phi$ to be a homotopy equivalence.

By Theorem 4.1, both $\|\Lambda\|$ and $|\Lambda|$ are contractible. So Theorem 3.4 follows directly from the following statement:

Theorem 5.3 There is a poset equivalence between $L_{n, k}$ and $|\Lambda|$. 
Lemma 5.4 Let $e$ be an edge in a $(n, k)$-graph $(\Gamma, \underline{l})$. Then there exists a maximal forest in $\Gamma-e$, that is a forest $\mathcal{F}$ such that $\Gamma / \mathcal{F}$ is a (reduced) $(n, k)$-graph.

Proof Let $\hat{\Gamma}$ be the graph of genus $n$ obtained from $\Gamma$ by collapsing the cycles. Take a maximal forest $\hat{\mathcal{F}}$ in $\hat{\Gamma}$. It can be extended to a maximal forest $\mathcal{F}$ of $\Gamma$ by picking all but one edge in each cycle.

If $e$ is an edge in a cycle $\mathbb{S}_{j}$, we can choose such a maximal forest containing $\mathbb{S}_{j}-\{e\}$ to obtain the result. If $e$ is not in a cycle, $e$ is non-separating in $\Gamma$ by assumption and hence non-separating in $\hat{\Gamma}$, so we can choose a forest $\hat{\mathcal{F}}$ in $\hat{\Gamma}$ which does not contain $e$ and extend it to $\Gamma$ as above.

Proof of the Theorem Let $(\Gamma, \phi)$ be a marked $(n, k)$-graph. Its underlying $(n, k)$-graph has $\mathbb{S}_{j}=\phi\left(C_{j}\right)$ with basepoint $\circ_{j}$, and let $w_{j}$ be the path from $\circ_{j}$ to $*$ in $\Gamma$, image of the stem of the $j$ th cycle in $G_{n, k}$, so that $\phi\left(y_{j}\right)=\bar{w}_{j} \star \mathbb{S}_{j} \star w_{j}$. Define a graph $\tilde{\Gamma}$ by attaching $\theta^{j}$ to $\circ_{j}$ in $\Gamma$ for each $j$ and define a marking $\tilde{\phi}: R_{n+3 k} \rightarrow \tilde{\Gamma}$ by

$$
\begin{aligned}
\tilde{\phi}\left(x_{i}\right) & =\phi\left(x_{i}\right) \\
\tilde{\phi}\left(y_{j}\right) & =\bar{w}_{j} \star \mathbb{S}_{j} \star w_{j} \\
\tilde{\phi}\left(u_{j}\right) & =\bar{w}_{j} \star e_{1}^{j} \star \bar{e}_{2}^{j} \star w_{j} \\
\tilde{\phi}\left(v_{j}\right) & =\bar{w}_{j} \star e_{2}^{j} \star \bar{e}_{3}^{j} \star w_{j} .
\end{aligned}
$$

This defines an inclusion of $L_{n, k}$ into $\|\Lambda\|$. Indeed, the poset minimal elements of $L_{n, k}$ are mapped to $\Lambda$, so their stars are in $\|\Lambda\|$.

To show that it maps into the smaller subcomplex $|\Lambda|$, we need to show that for each $(\Gamma, \phi)$ in $L_{n, k}$, the pair $(\tilde{\Gamma}, \tilde{\phi})$ is essential in $\Lambda$, that is for each edge $e$ of $\tilde{\Gamma}$, there is an invariant forest $\mathcal{F} \in \tilde{\Gamma}-e$ such that $\tilde{\Gamma} / \mathcal{F} \in \Lambda$. Note first that a maximal invariant forest of $\tilde{\Gamma}$ has to lie in $\Gamma$. If $e$ lies in $\theta^{j}$ for some $j$, choose a maximal forest $\mathcal{F}$ in $\Gamma$ such that $\Gamma / \mathcal{F}$ is a reduced $(n, k)$-graph and hence $\tilde{\Gamma} / \mathcal{F}$ is in $\Lambda$. On the other hand, if $e$ lies in $\Gamma$, we know that such a forest exists in $\Gamma / e$ by Lemma 5.4.

We claim that the image of $L_{n, k}$ equals $|\Lambda|$. To show this, we need to show that the marking of any graph in $|\Lambda|$ is induced by a marking from $G_{n, k}$ to the graph minus the theta subgraphs, that is we need to show how that the image of $y_{j}$ can be decomposed as a cycle with a stem for each $j$ with at most a point in the intersection of any two different cycles, and we need to show that the $u_{j}$ 's and $v_{j}$ 's are mapped accordingly. This is true for the poset minimal elements, i.e. the elements of $\Lambda$ : the cycles are the $\mathbb{S}_{j}$ 's with basepoint $\circ_{j}=*$ 
and with stem $w_{j}$. So we need to show that the cycle structure is carried by the admissible blow-ups (that is the blow-ups which stay within $|\Lambda|$ ).

Let $(\Gamma, \psi) \in|\Lambda|$ be a graph which has a cycle structure and let $\gamma$ be an ideal edge at a vertex $v$ of $\Gamma$ such that $\Gamma^{\gamma}$ is still in $|\Lambda|$. We want to show that $\Gamma^{\gamma}$ has a cycle structure.

Case 1 Suppose that $v \neq o_{j}$ for all $j$, so there is no $\theta^{j}$ attached to $v$. Each cycle has at most two edges ending at $v$. If $\gamma$ has either 0 or 2 half edges from $\mathbb{S}_{j}$ for each $j$ except for at most one $j$, then the induced marking on $\Gamma^{\gamma}$ still has embedded circles, with intersection possibly smaller than in $\Gamma$.

Suppose now that there is $j \neq j^{\prime}$ such that both $j$ and $j^{\prime}$ have exactly one half edge in $\gamma$. Then the induced cycles in $\Gamma^{\gamma}$ are such that $\mathbb{S}_{j} \cap \mathbb{S}_{j^{\prime}}=\gamma$, where $\gamma$ is now the new edge in $\Gamma^{\gamma}$. But there is no forest $\mathcal{F}$ in $\Gamma^{\gamma}-\{\gamma\}$ such that $\Gamma^{\gamma} / \mathcal{F}$ is in $\Lambda$. Indeed, if we do not collapse $\gamma$ again, the induced marking will have the $j$ th and $j^{\prime}$ th cycles intersecting in $\gamma$. Hence $\Gamma^{\gamma}$ is not in $|\Lambda|$.

Case 2 Suppose that there is at least one $j$ such that $v=o_{j}$. In particular, there is at least one $\theta$-graph attached to $v$. Note first that the equivariance conditions on $\gamma$ imply that at most one half edge of a graph $\theta^{j}$ may be in $\gamma$, and if there is such an edge, the whole $\theta$-graph is moved along the new edge $\gamma$ in $\Gamma^{\gamma}$. Moreover, by the same argument as in Case 1, their can be at most one $j$ such that $\mathbb{S}_{j}$ has exactly one half edge in $\gamma$.

Suppose that there is a $j$ such that $\mathbb{S}_{j}$ has two half edges in $\gamma$. Then $e_{i}^{j}$ must also be in $\gamma$ for some $i$, otherwise any forest collapse which does not collapse $\gamma$ will all induce a marking with $\bar{w}_{j} \star \gamma \star \mathbb{S}_{j} \star \bar{\gamma} \star w_{j}$ for $y_{j}$ but still $\bar{w}_{j} \star e_{1}^{j} \star \bar{e}_{2}^{j} \star w_{j}$ for $u_{j}$. Similarly, if a half edge $e_{i}^{j}$ is in $\gamma$ for some $j$, then the two half edges of $\mathbb{S}_{j}$ terminating at $v$ must also be in $\gamma$. This shows that a blow up cannot disconnect $\theta_{j}$ from $\mathbb{S}_{j}$.

Lastly, if there is a $j$ such that only one half edge is in $\gamma$, then the induced marking will move $\circ_{j}$ and prolong $w_{j}$ if there is also an $e_{i}^{j}$ in $\Gamma$.

\section{$6 \quad$ A space $L_{n}^{k}$ for the group $A_{n}^{k}$}

We recall from Section 2 that there is a central extension

$$
\mathbb{Z}^{k} \rightarrow A_{n, k} \rightarrow A_{n}^{k}
$$

where $A_{n}^{k}$ is a subgroup of $\operatorname{Aut}\left(F_{n+k}\right)$. To this group extension, corresponds a sequence of contractible spaces

$$
F_{n, k} \rightarrow L_{n, k} \rightarrow L_{n}^{k}
$$


where the spaces are realizations of posets, the maps are poset maps and $F_{n, k} \cong$ $\mathbb{R}^{k}$. We describe here the spaces $L_{n}^{k}$ and $F_{n, k}$.

As $A_{n}^{k}$ is a subgroup of $\operatorname{Aut}\left(F_{n+k}\right)$, a natural way to construct a contractible space $L_{n}^{k}$ with an action of $A_{n}^{k}$ with finite stabilizers and finite quotient, is to consider a subcomplex of Auter space $X_{n+k}$, as small as possible to get as good an approximation as we can of the vcd. In the same spirit as the construction of $L_{n, k}$, one can consider the essential realization $\left|\Lambda^{\prime}\right|$ of the initial segment $\Lambda^{\prime}$ of reduced graphs of minimal norm with respect to the $n$-tuple of cyclic words $\left(y_{1}, \ldots, y_{k}\right)$. We claim that $\left|\Lambda^{\prime}\right|$ is homeomorphic to the following space $L_{n}^{k}$ :

Definition 6.1 An $\left(\begin{array}{l}k \\ n\end{array}\right)$-graph $(\Gamma, \underline{l})$ is a graph $\Gamma$ of genus $(n+k)$ with vertices of valence at least 3 , except the basepoint which has valence at least 2 , with no separating edges, and equipped with embeddings $l_{j}: S^{1} \rightarrow \Gamma$ for $j=1, \ldots, k$ such that any two $\mathbb{S}_{j}:=l_{j}\left(S^{1}\right)$ meet in at most a point and the dual graph of the $\mathbb{S}_{j}$ 's is a forest.

A marked $\left(\begin{array}{l}n \\ k\end{array}\right)$-graph $(\Gamma, \phi)$ is a graph $\Gamma$ equipped with a pointed homotopy equivalence $\phi: G_{n, k} \rightarrow \Gamma$ such that $(\Gamma, \phi \circ \underline{\kappa})$ is an $\left(\begin{array}{l}n \\ k\end{array}\right)$-graph.

Two marked $\left(\begin{array}{l}n \\ k\end{array}\right)$-graphs $\left(\Gamma^{1}, \phi^{1}\right)$ and $\left(\Gamma^{2}, \phi^{2}\right)$ are equivalent if there is a graph isomorphism $\psi: \Gamma^{1} \rightarrow \Gamma^{2}$ such that $\psi \circ \phi^{1}\left(C_{j}\right)=\phi^{2}\left(C_{j}\right)$ and $\psi \circ \phi^{1} \simeq \phi^{2}$ (rel. $C_{j}$ for all $j$, up to a rotation).

Define now $L_{n}^{k}$ to be the poset of equivalence classes of marked $\left(\begin{array}{l}n \\ k\end{array}\right)$-graphs (or its realization) where the poset structure is given by the same admissible edge collapses as for $L_{n, k}$ (see p6).

Theorem 6.2 There is a poset equivalence $L_{n}^{k} \rightarrow\left|\Lambda^{\prime}\right|$ and hence $L_{n}^{k}$ is contractible.

The proof is totally analogous to the proof of Theorem [5.3 although a little bit simpler as we do not need to carry around $\Theta$-graphs. The poset map $L_{n}^{k} \rightarrow X_{n+k}$ takes the minimal poset elements of $L_{n}^{k}$ to $\Lambda^{\prime}$, and $L_{n}^{k}$ maps into the essential realization of $\Lambda^{\prime}$ by Lemma 5.4. On the other hand, we have $\Lambda^{\prime} \subset L_{n}^{k}$ and the inclusion of $\left|\Lambda^{\prime}\right|$ reduced to the case 1 in the proof of Theorem 5.3 Finally, $\left|\Lambda^{\prime}\right|$ is contractible by Theorem 4.1 with $G$ the trivial group.

Proposition 6.3 The quotient space $L_{n}^{k} / A_{n}^{k}:=Q L_{n}^{k}$ has dimension $2 n+2 k-2$ if $n \geq 1$ and dimension $k-1$ if $n=0$. 
Again, the proof is essentially the same as for $Q L_{n, k}$ (in Theorem 3.5).

Note that Collins constructed a space for $A_{0}^{k}$ which is isomorphic to our space $L_{0}^{k}$ (up to basepoint questions) [4. In this particular case when $n=0$, the dimension is equal to the vcd.

There is a forgetful map $L_{n, k} \rightarrow L_{n}^{k}$. Let $F_{n, k}$ be the fiber over the rose $\left(R_{n+k}, i d\right)$ with the $\mathbb{Z}^{k}$-action induced by the action of $A_{n, k}$ on $L_{n, k}$.

Proposition 6.4 There is a poset equivalence $F_{n, k} \rightarrow \mathbb{Z}^{k} \times(\mathbb{Z} / 2)^{k}$, where the poset structure on $\mathbb{Z}^{k} \times(\mathbb{Z} / 2)^{k}$ is defined by $(\bar{z}, \bar{p}) \leq\left(\bar{z}^{\prime}, \bar{p}^{\prime}\right)$ iff for all $i$ we have $p_{i}=p_{i}^{\prime} \quad$ and $\quad z_{i}=z_{i}^{\prime}$ or $p_{i}<p_{i}^{\prime} \quad$ and $\quad z_{i}=z_{i}^{\prime}$ or $z_{i}=z_{i}^{\prime}+1$.

Moreover, the realization of the poset $\mathbb{Z}^{k} \times(\mathbb{Z} / 2)^{k}$ is homeomorphic to $\mathbb{R}^{k}$ and the $\mathbb{Z}^{k}$-action on $F_{n, k}$ translates to the canonical $\mathbb{Z}^{k}$-action on $\mathbb{R}^{k}$.

Proof Suppose $(\Gamma, \phi) \in F_{n, k}$. Then $\Gamma$ is the rose $R_{n+k}$ with additional basepoints for the cycles, which are either equal to the basepoint of the graph, or a valence two vertex on the petal. Up to $(n, k)$-graph isomorphisms, there are $(\mathbb{Z} / 2)^{k}$ possibilities. We identify $\bar{p} \in(\mathbb{Z} / 2)^{k}$ with the rose which has a valence two vertex on the $(n+j)$ th petal if $p_{j}=1$ and no such vertex if $p_{j}=0$.

Now $\phi: G_{n, k} \rightarrow R_{n+k}$ is homotopic to the identity if the cycles are allowed to rotate. So there are $\mathbb{Z}^{k}$ possibilities for $\phi$, where $\bar{z} \in \mathbb{Z}^{k}$ for a rose $(R, \bar{p})$ represents the map $\phi$ which maps the cycles via the identity, according to the basepoints parametrized by $\bar{p}$, and wraps the $j$ th stem $z_{j}$ times around the $j$ th cycle.

If $p_{j}=0$ for all $j$, then the graph is reduced. If there exists a $j$ such that $p_{j}=1$, then there are two possible collapses on the $j$ th petal, one which leaves $z_{j}$ constant, and one which increases $z_{j}$ by 1 . (We choose the identification $\phi \rightsquigarrow \bar{z}$ so that this is the case.) This corresponds to the poset structure of $\mathbb{Z}^{k} \times(\mathbb{Z} / 2)^{k}$.

We are left to show that the realization of the poset $\mathbb{Z}^{k} \times(\mathbb{Z} / 2)^{k}$ is homeomorphic to $\mathbb{R}^{k}$ and that the induced $\mathbb{Z}^{k}$ action is the canonical action. Embed the set $\mathbb{Z}^{k} \times(\mathbb{Z} / 2)^{k}$ in $\mathbb{R}^{k}$ by mapping $(\bar{z}, \bar{p})$ to $\left(\bar{z}+\frac{\bar{p}}{2}\right)=\left(z_{1}+\frac{p_{1}}{2}, \ldots, z_{k}+\frac{p_{k}}{2}\right) \in \mathbb{R}^{k}$. Let $C_{\bar{z}}$ be the cube of length 1 in $\mathbb{R}^{k}$ with bottom corner $\bar{z}$ and top corner $\bar{z}+\overline{1}$. Let $c_{\bar{z}}=\left(z_{1}+\frac{1}{2}, \ldots, z_{k}+\frac{1}{2}\right)$ be the center of the cube. Then we have $C_{\bar{z}} \cap F_{n, k}=\left\{(\bar{z}, \bar{p}) \in F_{n, k} \mid(\bar{z}, \bar{p}) \leq c_{\bar{z}}=(\bar{z}, \overline{1})\right\}$. Similarly, the center of each face is the maximal element of the face in the poset. It follows that the realization of $\mathbb{Z}^{k} \times(\mathbb{Z} / 2)^{k}$ is $\mathbb{R}^{k}$. The $\mathbb{Z}^{k}$-action, which is by addition on the $\bar{z}$-coordinate, is then by translation. 
The contractibility of $L_{n}^{k}$ and $F_{n, k}$ leads to an alternative proof of the contractibility of $L_{n, k}$. This second approach, suggested to us by the referee, makes more apparent the fact that the $\Theta$-graphs in the original proof of the contractibility of $L_{n, k}$ only play the role of adding basepoints.

\section{Presentation}

We denote by $P_{i, j}$ the automorphism of the free group $F_{n}=\left\langle x_{1}, \ldots, x_{n}\right\rangle$ which permutes $x_{i}$ and $x_{j}$ and leaves the other generators fixed, and we denote by $I_{i}$ the automorphism that maps $x_{i}$ to its inverse and fixes the other generators.

For Whitehead moves, we use the following shortened notation: for $\epsilon, \eta= \pm 1$,

- $\left(z_{i}^{\epsilon} ; z_{j}^{\eta}\right)$ denotes the automorphism which maps $z_{i}$ to $z_{i} z_{j}^{\eta}$ if $\epsilon=1$ and to $z_{j}^{-\eta} z_{i}$ if $\epsilon=-1$, and fixes the other generators;

- $\left(z_{i}^{ \pm} ; z_{j}^{\eta}\right)$ denotes the automorphism which maps $z_{i}$ to $z_{j}^{-\eta} z_{i} z_{j}^{\eta}$ and fixes the other generators.

In terms of the discussion of Whitehead moves at the beginning of Section 4 before Theorem 4.1] $\left(z_{i}^{\epsilon} ; z_{j}^{\eta}\right):=\left(\left\{z_{i}^{\epsilon}, z_{j}^{\eta}\right\} ; z_{j}^{\eta}\right)$ and $\left(z_{i}^{ \pm} ; z_{j}^{\eta}\right):=\left(\left\{z_{i}, z_{i}^{-1}, z_{j}^{\eta}\right\} ; z_{j}^{\eta}\right)$.

Note that $\left(z_{i}^{\epsilon} ; z_{j}^{\eta}\right)^{-1}=\left(z_{i}^{\epsilon} ; z_{j}^{-\eta}\right)$ and $\left(z_{i}^{ \pm} ; z_{j}^{\eta}\right)^{-1}=\left(z_{i}^{ \pm} ; z_{j}^{-\eta}\right)$. We will use the second notation when writing relations for simplicity.

Theorem 7.1 The following gives a presentation for $\mathrm{A}_{n}^{k}$ :

Generators:

$$
\begin{array}{ll}
P_{i, j} & \text { for } 1 \leq i, j \leq n \text { and } i \neq j \\
I_{i} & \text { for } 1 \leq i \leq n \\
\left(x_{i}^{\epsilon} ; z\right) & \text { for } 1 \leq i \leq n, \epsilon= \pm 1 \text { and } x_{i} \neq z \in\left\{x_{1}, \ldots, x_{n}, y_{1}, \ldots, y_{k}\right\} \\
\left(y_{i}^{ \pm} ; z\right) & \text { for } 1 \leq i \leq k \text { and } y_{i} \neq z \in\left\{x_{1}, \ldots, x_{n}, y_{1}, \ldots, y_{k}\right\}
\end{array}
$$

Relations: For $z, z_{i} \in\left\{x_{1}, \ldots, x_{n}, y_{1}, \ldots, y_{k}\right\}$ and $w, w_{i}=x_{j_{i}}^{\delta_{i}}$ or $y_{j_{i}}^{ \pm}$,

Q1 Relations in $\operatorname{Aut}\left(F_{n}\right)$ for $\left\{\left(x_{i}^{\epsilon} ; x_{j}\right), P_{i, j}, I_{j}\right\}$

Q2 $\left(w_{1} ; z_{1}\right)\left(w_{2} ; z_{2}\right)=\left(w_{2} ; z_{2}\right)\left(w_{1} ; z_{1}\right) \quad$ for $w_{1} \neq w_{2}$ and $z_{i}^{ \pm 1} \notin\left\{w_{1}, w_{2}\right\}$

Q3 (1) $\left(y_{i}^{ \pm} ; x_{j}\right) P_{j, l}=P_{j, l}\left(y_{i}^{ \pm} ; x_{l}\right)$

(2) $\left(y_{i}^{ \pm} ; x_{j}\right) I_{j}=I_{j}\left(y_{i}^{ \pm} ; x_{j}^{-1}\right)$

Q4 (1) $\left(w ; x_{j}^{\eta}\right)\left(x_{j}^{\eta} ; z\right)\left(w ; x_{j}^{-\eta}\right)=(w ; z)\left(x_{j}^{\eta} ; z\right)$

(2) $\left(y_{i}^{ \pm} ; z^{\epsilon}\right)\left(w ; y_{i}\right)\left(y_{i}^{ \pm} ; z^{-\epsilon}\right)=\left(w ; z^{-\epsilon}\right)\left(w ; y_{i}\right)\left(w ; z^{\epsilon}\right)$

Q5 $\quad\left(y_{i}^{ \pm} ; x_{j}^{\eta}\right)\left(x_{j}^{-\eta} ; y_{i}\right)=\left(x_{j}^{\eta} ; y_{i}^{-1}\right)\left(y_{i}^{ \pm} ; x_{j}^{\eta}\right)$

whenever these symbols denote generators or their inverses. 
In particular, for $n=0$ we recover McCool's presentation of the pure symmetric (or basis-conjugating) automorphisms of free groups [25], which has generators $\left\{\left(y_{i}^{ \pm} ; y_{j}\right) \mid 1 \leq i, j \leq k, i \neq j\right\}$ and relations

$$
\begin{array}{ll}
\text { Z1 } & \left(y_{i}^{ \pm} ; y_{j}\right)\left(y_{l}^{ \pm} ; y_{j}\right)=\left(y_{l}^{ \pm} ; y_{j}\right)\left(y_{i}^{ \pm} ; y_{j}\right) \\
\text { Z2 } & \left(y_{i}^{ \pm} ; y_{j}\right)\left(y_{l}^{ \pm} ; y_{m}\right)=\left(y_{l}^{ \pm} ; y_{m}\right)\left(y_{i}^{ \pm} ; y_{j}\right) \\
\text { Z3 } & \left(y_{i}^{ \pm} ; y_{j}\right)\left(y_{l}^{ \pm} ; y_{j}\right)\left(y_{i}^{ \pm} ; y_{l}\right)=\left(y_{i}^{ \pm} ; y_{l}\right)\left(y_{i}^{ \pm} ; y_{j}\right)\left(y_{l}^{ \pm} ; y_{j}\right)
\end{array}
$$

where the indices are assumed to be different when given by different letters.

Theorem 7.2 The following gives a presentation for $\mathrm{A}_{n, k}$ :

Generators: same as for $\mathrm{A}_{n}^{k}$ with $k$ additional generators $\left(y_{i}^{ \pm} ; y_{i}\right)$ for $1 \leq i \leq k$.

Relations: Q1, Q2, Q3, Q4 (never allowing a symbol $\left(y_{i}^{ \pm} ; y_{i}\right)$ ), and

$$
\begin{array}{ll}
Q 2^{\prime} & \left(y_{i}^{ \pm} ; y_{i}\right) a=a\left(y_{i}^{ \pm} ; y_{i}\right) \quad \text { with } a=P_{i, j}, I_{j} \text { or }(w ; z) \\
Q 5^{\prime} & \left(y_{i}^{ \pm} ; x_{j}^{\eta}\right)\left(x_{j}^{-\eta} ; y_{i}\right)\left(y_{i}^{ \pm} ; x_{j}^{-\eta}\right)\left(x_{j}^{\eta} ; y_{i}\right)=\left(y_{i}^{ \pm} ; y_{i}^{-1}\right)
\end{array}
$$

whenever these symbols denote generators or their inverses.

The generator $\left(y_{i}^{ \pm} ; y_{i}\right):=\left(x_{1}, \ldots, x_{n}, 1, \ldots, y_{i}, \ldots, 1\right) \in \mathrm{A}_{n, k}$ should be thought of as the conjugation of $y_{i}$ by itself, even though this is trivial as an automorphism of the free group.

Proof of Theorem 7.1 The group $\operatorname{Aut}\left(F_{n+k}\right)$ acts on the set of cyclic words with letters in $L_{n+k}:=\left\{x_{1}, x_{1}^{-1}, \ldots, y_{k}, y_{k}^{-1}\right\}$ and $\mathrm{A}_{n}^{k}$ is the stabilizer of the $k$-tuple of cyclic words $U=\left(y_{1}, \ldots, y_{k}\right)$. Theorem 1.1 in 24] says that such a group is finitely presented. As remarked in [24, Section 4(1)], the proof actually constructs a finite 2-dimensional complex $K$ whose fundamental group is this stabilizer and hence gives a presentation. We analyze this complex.

The vertices of the complex in our case are

$$
K_{0}:=\left\{V \text { minimal tuple } \mid V=U P \text { for some } P \in \operatorname{Aut}\left(F_{n+k}\right)\right\}=\left\{U \sigma \mid \sigma \in \Omega_{k}\right\}
$$

where a minimal tuple is a tuple of reduced cyclic words of minimal length in the orbit of $U$ and $\Omega_{k} \cong \Sigma_{k}\left\langle\mathbb{Z}_{2}\right.$ is the subgroup of $\operatorname{Aut}\left(F_{n+k}\right)$ of permutations and inverses of the $y_{i}$ 's. So $K_{0} \cong \Omega_{k}$.

Let $\mathcal{W}_{n+k}$ denote the set of Whitehead automorphisms for $\operatorname{Aut}\left(F_{n+k}\right)$. We recall that $\mathcal{W}_{n+k}$ is the finite set $T_{1} \cup T_{2}$ where $T_{1}=\Omega_{n+k}$ is the set of permutations and inverses and $T_{2}=\left\{(A ; a) \mid A \subset L_{n+k}, a \in A\right.$ and $\left.a^{-1} \notin A\right\}$ with $(A ; a)$ denoting the automorphism mapping $z \in L_{n+k}$ to $z a$ (and $z^{-1}$ to 
$a^{-1} z^{-1}$ ) if $a \neq z \in A$ and $z^{-1} \notin A$, mapping $z$ to $a^{-1} z a$ (and $z^{-1}$ to $a^{-1} z^{-1} a$ ) if $z, z^{-1} \in A$ and fixing $z$ otherwise. The set of edges in the complex is

$$
K_{1}:=\left\{\left(V_{1}, V_{2} ; P\right) \mid P \in \mathcal{W}_{n+k} \text { and } V_{1} P=V_{2}\right\} / \sim .
$$

Note that if $P \in \mathcal{W}$, then $P^{-1}$ is also in $\mathcal{W}$. The directed edge $e=\left(V_{1}, V_{2} ; P\right)$, from $V_{1}$ to $V_{2}$, is identified with $\bar{e}=\left(V_{2}, V_{1} ; P^{-1}\right)$ in $K_{1}$. Finally the 2-cells are all the cells coming from the relations $\mathrm{R} 1$ to $\mathrm{R} 10$ in [23], attached to directed paths. We recall these relations below.

There are two types of edges in $K$, coming from the two types of Whitehead automorphisms:

Type I edges $(U \sigma, U \sigma \tau ; \gamma)$ for $\sigma, \tau \in \Omega_{k}$ and $\gamma \in \Omega_{n+k}$ such that $(U \sigma) \gamma=$ $U \sigma \tau$. In particular, $\gamma$ is only allowed to permute the $y_{i}$ 's among themselves. Hence $\gamma \in \Omega_{n} \times \Omega_{k} \subset \Omega_{n+k}$ and $\gamma=(\bar{\gamma}, \tau)$. So the set of type I edges between any two vertices is isomorphic to $\Omega_{n}$.

Type II edges Type II edges do not involve permutations so they can exist only from one vertex to itself in our complex. Moreover, they have to preserve the cyclic words $y_{1}, \ldots, y_{k}$, so they have the form

$$
\left(U \sigma, U \sigma ;\left(X \sqcup\left\{y_{i_{1}}, y_{i_{1}}^{-1}, \ldots, y_{i_{r}}, y_{i_{r}}^{-1}\right\} \sqcup\{a\} ; a\right)\right.
$$

where $X \subset L_{x}=\left\{x_{1}, x_{1}^{-1}, \ldots, x_{n}, x_{n}^{-1}\right\}$ and $a \in L_{n+k}$.

Choose a maximal tree $T$ in $K_{1}$ with edges of type $(U \sigma, U \sigma \tau ; \tau)$ for some $\tau \in \Omega_{k} \cong\{1\} \times \Omega_{k}$. The generators of $\pi_{1} K \cong \mathrm{A}_{n}^{k}$ are given by the edges in $K_{1}-T$.

If we consider the subcomplex of $K$ formed by taking all the edges of the form $(U \sigma, U \sigma \tau ; \tau)$ for $\tau \in \Omega_{k}$ and the 2-cells given by the relations R7 (which is a set of relations for $\Omega_{n+k}$ ) restricted to these edges, we obtain a retraction of the 2-skeleton of $E \Omega_{k}$, which is simply connected. It follows that all edges of type $(U \sigma, U \sigma \tau ; \tau)$ give trivial generators in $\mathrm{A}_{n}^{k}$.

Moreover, $(U \sigma, U \sigma \tau ;(\gamma, \tau)) \sim(U, U ;(\gamma, 1))$ as $(1, \sigma)(\gamma, \tau)=(\gamma, 1)(1, \sigma \tau)$ in $\Omega_{n+k}$ (giving thus a relation in $\left.\mathrm{R} 7\right)$ and $(U \sigma, U \sigma ;(A ; a)) \sim\left(U, U ;\left(A \sigma^{-1} ; a \sigma^{-1}\right)\right)$ as $(1, \sigma)(A ; a)=\left(A \sigma^{-1} ; a \sigma^{-1}\right)(1, \sigma)$ by R6. (R6 says that $T^{-1}(A ; a) T=$ $(A T ; a T)$ for any $T \in T_{1}$.) So the loops at the vertex $U$ generate the group. Also, the relations given by 2 -cells in the complex all follow from the relations R1-R10 interpreted on the generators $(U, U ; \gamma)$ and $(U, U ;(A ; a))$. Indeed, the other relations are conjugated to these (except for the relations in R6 and R7 already used to identify generators) and thus do not bring anything new by R6, used in the case $T=(1, \sigma)$. 
$\mathrm{R} 1$ identifies the inverses in $T_{2}$ and $\mathrm{R} 2$ says that $(A ; a)(B ; a)=(A \cup B ; a)$ when $A \cap B=\{a\}$, showing thus that the generators we propose are indeed generators for the group. Note that R2 also implies the commutation relations of Q2 when $z_{1}=z_{2}$. As the relations in the Theorem are easily verified, we are left to show that they imply the relations R3-R10.

$\mathrm{R} 3$ is a set of commutation relations and follows from Q2 when $z_{1} \neq z_{2}$.

R4 says that $(B ; b)^{-1}(A ; a)(B ; b)=(A+B-b ; a)$ when $A \cap B=\emptyset, a^{-1} \notin B$ and $b^{-1} \in A$. We need to deduce it from our relations when $(A ; a)$ and $(B ; b)$ define loops at $U$ in $K$. In particular, we need $b=x_{i}^{\epsilon} \in L_{x}$ as $b^{-1} \in A$ but $b \notin A$ and $b \neq a^{-1}$. Using Q2, we can isolate a minimal case on the left hand side: $\left(B-w ; x_{i}^{\epsilon}\right)^{-1}\left(w ; x_{i}^{-\epsilon}\right)\left(x_{i}^{-\epsilon} ; a\right)\left(w ; x_{i}^{\epsilon}\right)\left(A-x_{i}^{-\epsilon} ; a\right)\left(B-w ; x_{i}^{\epsilon}\right)$. We then use Q4 (1) and repeat the procedure with all the elements of $B$.

R5 says that $(A ; a)\left(A-a+a^{-1} ; b\right)=\left(\begin{array}{cc}a & b \\ \downarrow & \downarrow \\ b^{-1} & a\end{array}\right)\left(A-b+b^{-1} ; a\right)$ when $b \in A$, $b^{-1} \notin A$ and $a \neq b$. In our case, we need to have $a, b \in L_{x}$. The relation follows from Q1 when only $x$ 's are involved. Using R2, we isolate $(b ; a)\left(a^{-1} ; b\right)$ in the left hand side and use R5 in this minimal case (that is use Q1). We then use Q3 to move the matrix (element of T1) in front of the left hand side and finally use R4.

R6, which was recalled above, follows from Q1 and Q3 (using Q2 as usual).

$\mathrm{R} 7$ gives a set of relations for $\Omega_{n}$, which is included in Q1.

R8 says that $(A ; a)=\left(L_{n+k}-a^{-1} ; a\right)\left(L_{n+k}-A ; a^{-1}\right)=\left(L_{n+k}-A ; a^{-1}\right)\left(L_{n+k}-\right.$ $\left.a^{-1} ; a\right)$. This just follows from R1-R2.

R9 says that $\left(L_{n+k}-b ; b^{-1}\right)(A ; a)\left(L_{n+k}-b^{-1} ; b\right)=(A ; a)$ if $b, b^{-1} \notin A$. In particular, $a \neq b$. This relation follows from Q4. Indeed, if $a=x_{j}^{\delta} \in L_{x}$, isolate $\left(x_{j}^{-\delta} ; b^{-1}\right)$ from of the left hand side and use Q4 (1) to pass it over $\left(A ; x_{j}^{\delta}\right)$, which gives $\left(L_{n+k}-b-x_{j}^{-\delta} ; b^{-1}\right)\left(A ; x_{j}^{\delta}\right)\left(A-x_{j}^{\delta}+b^{-1} ; b^{-1}\right)\left(x_{j}^{-\delta} ; b^{-1}\right)\left(L_{n+k}-b^{-1} ; b\right)$.

Now simplify and isolate $\left(x_{j}^{\delta} ; b\right)$ and use again Q4(1) to pass it over $\left(A ; x_{j}^{\delta}\right)$, which gives $\left(L_{n+k}-b-x_{j}^{-\delta} ; b^{-1}\right)\left(x_{j}^{\delta} ; b\right)\left(A-x_{j}^{\delta}+b ; b\right)\left(A ; x_{j}^{\delta}\right)\left(L_{n+k}-A-x_{j}^{-\delta}-\right.$ $\left.b^{-1} ; b\right)$.

This simplifies to $\left(A ; x_{j}^{\delta}\right)$ as required.

If on the other hand $a=y_{i}^{\epsilon} \in L_{y}$, isolating $\left(y_{i}^{ \pm} ; b^{-1}\right)$ in the left hand side and using Q4 $(2)$ to pass it over $\left(A ; y_{i}^{\epsilon}\right)$ gives 
$\left(L_{n+k}-b-y_{i}^{ \pm} ; b^{-1}\right)\left(A-y_{i}^{\epsilon}+b ; b\right)\left(A ; y_{i}^{\epsilon}\right)\left(A-y_{i}^{\epsilon}+b^{-1} ; b^{-1}\right)\left(y_{i}^{ \pm} ; b^{-1}\right)\left(L_{n+k}-b^{-1} ; b\right)$, which simplifies to $\left(A ; y_{i}^{\epsilon}\right)$.

R10 says that $\left(L_{n+k}-b ; b^{-1}\right)(A ; a)\left(L_{n+k}-b^{-1} ; b\right)=\left(L_{n+k}-A ; a^{-1}\right)$ if $b \neq a$, $b \in A$ and $b^{-1} \notin A$. So $b \in L_{x}$ in our case. If $a \in L_{x}$, we can follow [23] and show that the relation follows from R9 and R5. We do the case $a \in L_{y}$. So we take $a=y_{j}^{\eta}, b=x_{i}^{\epsilon}$ The left hand side in the equation equals

$$
\left(L_{n+k}-x_{i}^{\epsilon}-y_{j}^{ \pm} ; x_{i}^{-\epsilon}\right)\left(y_{j}^{ \pm} ; x_{i}^{-\epsilon}\right)\left(x_{i}^{\epsilon} ; y_{j}^{\eta}\right)\left(A-x_{i}^{\epsilon} ; y_{j}^{\eta}\right)\left(L_{n+k}-x_{i}^{-\epsilon} ; x_{i}^{\epsilon}\right)
$$

which, using Q5, gives

$$
\left(L_{n+k}-x_{i}^{\epsilon}-y_{j}^{ \pm} ; x_{i}^{-\epsilon}\right)\left(x_{i}^{-\epsilon} ; y_{j}^{-\eta}\right)\left(y_{j}^{ \pm} ; x_{i}^{-\epsilon}\right)\left(A-x_{i}^{\epsilon} ; y_{j}^{\eta}\right)\left(L_{n+k}-x_{i}^{-\epsilon} ; x_{i}^{\epsilon}\right) \text {. }
$$

Now we use Q4 (1) to pass $\left(x_{i}^{-\epsilon} ; y_{j}^{-\eta}\right)$ to the left, and Q4(2) to pass $\left(y_{j}^{ \pm} ; x_{i}^{-\epsilon}\right)$ over $\left(A-x_{i}^{\epsilon} ; y_{j}^{\eta}\right)$, which gives

$\left(x_{i}^{-\epsilon} ; y_{j}^{-\eta}\right)\left(L_{n+k}-x_{i}^{\epsilon}-y_{j}^{\eta} ; y_{j}^{-\eta}\right)\left(L_{n+k}-x_{i}^{\epsilon}-y_{j}^{ \pm} ; x_{i}^{-\epsilon}\right)\left(A-y_{j}^{\eta} ; x_{i}^{\epsilon}\right)\left(A-x_{i}^{\epsilon} ; y_{j}^{\eta}\right)$ $\left(A-x_{i}^{\epsilon}-y_{j}^{\eta}+x_{i}^{-\epsilon} ; x_{i}^{-\epsilon}\right)\left(y_{j}^{ \pm} ; x_{i}^{-\epsilon}\right)\left(L_{n+k}-x_{i}^{-\epsilon} ; x_{i}^{\epsilon}\right)$.

This simplifies to $\left(L_{n+k}-A ; y_{j}^{-\eta}\right)$ as required.

To prove Theorem 7.2 we need a lemma about group extensions.

Let $1 \rightarrow A \stackrel{i}{\rightarrow} E \stackrel{\pi}{\rightarrow} G \rightarrow 1$ be a central extension of a group $G$ by an abelian group $A$, with a section of sets $s: G \rightarrow E$ (so $\pi \circ s=i d$ ) satisfying $s(1)=1$ and $f: G \times G \rightarrow A$ the classifying cocycle of the extension, defined by

$$
s(g) s(h)=i \circ f(g, h) s(g h) .
$$

Note that if $r_{1} \ldots r_{m}=1$ in $G$, then $s\left(r_{1} \ldots r_{m}\right)=1$ in $E$ and hence

$$
s\left(r_{1}\right) \ldots s\left(r_{m}\right)=f\left(r_{1}, r_{2}\right) f\left(r_{1} r_{2}, r_{3}\right) \ldots f\left(r_{1} \ldots r_{m-1}, r_{m}\right) .
$$

We denote by $s^{f}$ the operator lifting the relations of $G$ to relations in $E$ this way.

Lemma 7.3 Let $1 \rightarrow A \stackrel{i}{\rightarrow} E \stackrel{\pi}{\rightarrow} G \rightarrow 1$ be a central extension as above. If $A$ and $G$ are finitely presented, with presentation

$$
\begin{aligned}
A & =\left\langle a_{1}, \ldots, a_{k}\right\rangle / R_{A} \\
G & =\left\langle g_{1}, \ldots, g_{n}\right\rangle / R_{G} .
\end{aligned}
$$

Then $E$ is finitely presented, with presentation

$$
E=\left\langle i\left(a_{1}\right) \ldots, i\left(a_{k}\right), s\left(g_{1}\right), \ldots, s\left(g_{n}\right)\right\rangle /[A, G] \cup i\left(R_{A}\right) \cup s^{f}\left(R_{G}\right)
$$

where $[A, G]$ is the set of commutation relations $i\left(a_{j}\right) s\left(g_{i}\right)=s\left(g_{i}\right) i\left(a_{j}\right)$ and $s^{f}\left(R_{G}\right)$ is the lift of the relations $R_{G}$ to $E$ twisted by $f$ as defined above. 
Proof Consider the free group $F:=\left\langle i\left(a_{1}\right) \ldots, i\left(a_{k}\right), s\left(g_{1}\right), \ldots, s\left(g_{n}\right)\right\rangle$. The natural map $h: F \rightarrow E$ is surjective as $i \times s: A \times G \rightarrow E$ is a bijection of sets (since $s(G)$ is a set of coset representatives). The relations $[A, G], i\left(R_{A}\right)$ and $s^{f}\left(R_{G}\right)$ are clearly satisfied in $E$, and so define elements in the kernel of $h$.

Let $e=i\left(a_{j_{1}}\right) \ldots i\left(a_{j_{p}}\right) s\left(g_{i_{1}}\right) \ldots s\left(g_{i_{q}}\right) \in F$ and suppose that $h(e)=1$. (It is enough to consider this case as $[A, G]$ is satisfied in $E$.) Then $\pi \circ h(e)=1$ in $G$, that is $g_{i_{1}} \ldots g_{i_{q}}=1$. This relation in $G$ lifts to a relation $s\left(g_{i_{1}}\right) \ldots s\left(g_{i_{q}}\right)=i(a)$ in $E$ for some $a \in A$. So $i\left(a_{j_{1}}\right) \ldots i\left(a_{j_{p}}\right) s\left(g_{i_{1}}\right) \ldots s\left(g_{i_{q}}\right)=i\left(a_{j_{1}}\right) \ldots i\left(a_{j_{p}}\right) i(a)$. As $h(e)=1$, we have $a_{j_{1}} \ldots a_{j_{p}} a=1$ is a relation in $A$ (as $i$ is an injective homomorphism). So the relation $h(e)=1$ follows from a relation in $s^{f}\left(R_{G}\right)$ and a relation in $i\left(R_{A}\right)$.

Proof of Theorem [7.2 There is a central extension $1 \rightarrow \mathbb{Z}^{k} \rightarrow \mathrm{A}_{n, k} \rightarrow$ $\mathrm{A}_{n}^{k} \rightarrow 1$. By the Lemma, the generators of $\mathrm{A}_{n, k}$ are the union of the generators of $\mathrm{A}_{n}^{k}$ with those of $\mathbb{Z}^{k}$. The last generators are denoted $\left(y_{i}^{ \pm} ; y_{i}\right)$, for $1 \leq i \leq k$, in the Theorem. Then relations for $\mathrm{A}_{n, k}$ are given by the relations for $\mathbb{Z}^{k}$, the commutations $\left[\mathbb{Z}^{k}, \mathrm{~A}_{n}^{k}\right]$ and the lifts of the relations from $\mathrm{A}_{n}^{k}$. Q2' takes care of the first two sets of relations and Q1,Q2,Q3,Q4 and Q5' is the lift of the relations from $\mathrm{A}_{n}^{k}$.

In [3], the group $\mathrm{A}_{0}^{k}$ is studied (denoted $C_{k}$ in the paper). They show that

$$
H_{1}\left(\mathrm{~A}_{0}^{k}\right)=\bigoplus_{1 \leq i, j \leq k, i \neq j} \mathbb{Z}\left[y_{i}^{ \pm} ; y_{j}\right]
$$

where $\mathbb{Z}\left[y_{i}^{ \pm} ; y_{j}\right]$ is the free abelian group generated by $\left[y_{i}^{ \pm} ; y_{j}\right]$. Recall that $\mathrm{A}_{0}^{k}$ can be thought of as an extended pure braid group (which is the mapping class group of a punctured sphere). Note in particular that, as for the pure braid group, $H_{1}$ is not stable with respect to $k$. The presentation given above allows us to calculate $H_{1}$ in the higher genus cases.

Theorem 7.4 $H_{1}\left(\mathrm{~A}_{n, k}\right) \cong \mathbb{Z}_{2} \cong H_{1}\left(\mathrm{~A}_{n}^{k}\right)$ if $n>2$

Proof If $n>2$, we have $H_{1}\left(\operatorname{Aut}\left(F_{n}\right)\right)=\mathbb{Z}_{2}$ generated by $\left[P_{i, j}\right]=\left[I_{k}\right]$ (the generators $\left(x_{i}^{\delta} ; x_{j}\right)$ being trivial in the abelianization).

For $\mathrm{A}_{n}^{k}, \mathrm{Q} 4(1)$ implies that $(w ; z)$ is trivial in the abelianization for any $w=x_{i}^{\delta}$ or $y_{i}^{ \pm}$and $z \in\left\{x_{1}, x_{2}, \ldots, y_{k}\right\}$. The only relations involving $P_{i, j}$ or $I_{k}$ which are not in $\operatorname{Aut}\left(F_{n}\right)$ give trivial relations in the abelianization, hence $H_{1}\left(\mathrm{~A}_{n}^{k}\right)=\mathbb{Z}_{2}$.

For $\mathrm{A}_{n, k}$, the additional relation $\mathrm{Q} 5$ ' implies that the generators $\left(y_{i}^{ \pm} ; y_{i}\right)$ are also trivial in the abelianization and hence we get the same result. 


\section{References}

[1] N. Brady, J. McCammond, J. Meier, and A. Miller, The pure symmetric automorphisms of a free group form a duality group, J. Algebra 246 (2001) 881-896.

[2] K. Brown, Cohomology of Groups, Springer-Verlag, New York-Berlin, 1982.

[3] A. Brownstein and R. Lee, Cohomology of the group of motions of $n$ strings in 3-space, Contemp. Math. vol. 150 (1993) 51-61.

[4] D. J. Collins, Cohomological dimension and symmetric automorphisms of a free group, Comment. Math. Helv. 64 (1989) 44-61.

[5] M. Culler and K. Vogtmann, Moduli spaces of graphs and automorphisms of free groups, Invent. Math. 84 (1986) 91-119.

[6] D. Dahm, A generalization of braid theory, Ph.D. Thesis, Princeton Univ., 1962.

[7] D. Goldsmith, The theory of motion groups, Mich. Math. J. 28 (1981), 3-17.

[8] J. L. Harer, Stability of the homology of the mapping class groups of orientable surfaces, Annals Math. 121 (1985), 215-249.

[9] A. Hatcher, Algebraic Topology, Cambridge University Press, 2002.

[10] A. Hatcher and K. Vogtmann, Cerf theory for graphs, J. London Math. Soc. (2) 58 (1998) 633-655.

[11] A. Hatcher and N. Wahl, Stabilization for the automorphisms of free groups with boundary, preprint, arXiv:math.GT/0406277

[12] C. A. Jensen, Cohomology of $\operatorname{Aut}\left(F_{n}\right)$ in the p-rank two case, J. Pure Appl. Algebra 158 (2001) 41-81.

[13] C. A. Jensen, Contractibility of fixed point sets of auter space, Topology Appl. 119 (2002) 287-304.

[14] C. A. Jensen, J. McCammond and J. Meier, The Euler characteristic of the Whitehead automorphism group of a free product, preprint.

[15] C. A. Jensen, Homology of holomorphs of free groups, J. Algebra 271 (2004) 281-294.

[16] S. Krstić, Actions of finite groups on graphs and related automorphisms of free groups, J. Algebra 124 (1989) 119-138.

[17] S. Krstić and K. Vogtmann, Equivariant outer space and automorphisms of free-by-finite groups, Comment. Math. Helv. 68 (1993) 216-262.

[18] G. Levitt, Automorphisms of hyperbolic groups and graphs of groups, preprint, arXiv:math.GR/0212088

[19] I. Madsen and U. Tillmann, The stable mapping class group and $\mathcal{Q}\left(\mathbb{C} P_{+}^{\infty}\right)$, Invent. math. 145 (2001), 509-544.

[20] I. Madsen and M. Weiss, The stable moduli space of Riemann surfaces: Mumford's conjecture, preprint, arXiv:math.AT/0212321 
[21] W. Magnus, A. Karrass and D. Solitar, Combinatorial group theory. Presentations of groups in terms of generators and relations, Second edition, Dover Pub., New York, 1976.

[22] J. McCammond and J. Meier, The hypertree poset and the $L^{2}$ Betti numbers of the motion group of the trivial link, Math. Ann. 328 (2004) 633-652.

[23] J. McCool, A presentation for the automorphism group of a free group of finite rank, J. London Math. Soc. (2) 8 (1974),259-266.

[24] J. McCool, Some finitely presented subgroups of the automorphism group of a free group, J. Algebra 35 (1975), 205-213.

[25] J. McCool, On basis-conjugating automorphisms of the free groups, Can. J. Math., 38 (1986) no. 6, 1525-1529.

[26] D. McCullough and A. Miller, Symmetric Automorphisms of Free Products, Mem. Amer. Math. Soc. 122 (1996), no. 582.

[27] D. Quillen, Homotopy properties of the poset of non-trivial p-subgroups of a group, Advances in Math. 28 (1978) 101-128.

[28] U. Tillmann, On the homotopy of the stable mapping class group, Invent. Math. 130 (1997), 257-275.

[29] N. Wahl, From mapping class groups to automorphisms of free groups, preprint, arXiv:math.AT/0406278

Department of Mathematics, University of New Orleans

New Orleans, LA 70148, USA

and

Department of Mathematics, University of Aarhus

Ny Munkegade 116, 8000 Aarhus, DENMARK

Email: jensen@math.uno.edu and wahl@imf.au.dk

Received: 26 February 2004 Revised: 8 July 2004 